\title{
Testes bayesianos para \\ homogeneidade marginal em tabelas de contingência
}

Helton Graziadei de Carvalho

\author{
DissertaÇÃo APRESEnTADA \\ $\mathrm{AO}$ \\ Instituto DE Matemática E EstatísticA \\ DA \\ Universidade De SÃo Paulo \\ PARA \\ OBTENÇÃO DO TÍTULO \\ $\mathrm{DE}$ \\ Mestre em CiênCias
}

\author{
Programa: Estatística \\ Orientador: Prof. Dr. Luís Gustavo Esteves
}

Durante o desenvolvimento deste trabalho o autor recebeu auxílio financeiro do CNPq

São Paulo, agosto de 2015 


\section{Testes bayesianos para homogeneidade marginal em tabelas de contingência}

Esta versão da dissertação contém as correções e alterações sugeridas pela Comissão Julgadora durante a defesa da versão original do trabalho, realizada em 06/08/2015. Uma cópia da versão original está disponível no Instituto de Matemática e Estatística da Universidade de São Paulo.

Comissão Julgadora:

- Prof. Dr. Luís Gustavo Esteves (orientador) - IME-USP

- Prof. Dr. Carlos Alberto de Bragança Pereira - IME-USP

- Prof. Dr. Rafael Izbicki - DEs-UFSCar 
"De tudo, ficaram três coisas:

A certeza de que estamos começando, a certeza de que é preciso continuar e a certeza de que podemos ser interrompidos antes de terminar.

Façamos da interrupção um caminho novo, da queda um passo de dança, do medo uma escada, do sono uma ponte, da procura um encontro." - Fernando Sabino 


\section{Agradecimentos}

Primeiramente agradeço aos meus pais, que sempre me proporcionaram todo o apoio emocional e financeiro necessário ao longo dessa jornada. Ao meu irmão pela companhia, incentivo e por me auxiliar bastante neste período em São Paulo. À minha tia pelos conselhos e pela paciência ao longo de todos esses anos.

Ao meu orientador, Professor Luís Gustavo Esteves, por sua dedicação constante, paciência inesgotável e apoio incondicional.

Aos Professores Carlos A. B. Pereira e Sergio Wechsler pelos ensinamentos valiosos, além da disposição em auxiliar no que fosse necessário para aprimorar minha formação. Ao Professor Marcelo Lauretto pela paciência e ajuda na implementação das rotinas. Aos Professores Mário de Castro e Cibele M. Russo por terem me estimulado a continuar na carreira acadêmica.

À todos os meus amigos que, de alguma forma, contribuíram para o desenvolvimento desta dissertação. 


\section{Resumo}

CARVALHO, H.G. Testes bayesianos para homogeneidade marginal em tabelas de contingência. 2015. 65 f. Dissertação (Mestrado) - Instituto de Matemática e Estatística, Universidade de São Paulo, São Paulo, 2015.

O problema de testar hipóteses sobre proporções marginais de uma tabela de contingência assume papel fundamental, por exemplo, na investigação da mudança de opinião e comportamento. Apesar disso, a maioria dos textos na literatura abordam procedimentos para populações independentes, como o teste de homogeneidade de proporções. Existem alguns trabalhos que exploram testes de hipóteses em caso de respostas dependentes como, por exemplo, o teste de McNemar para tabelas $2 \times 2$. A extensão desse teste para tabelas $k \times k$, denominado teste de homogeneidade marginal, usualmente requer, sob a abordagem clássica, a utilização de aproximações assintóticas. Contudo, quando o tamanho amostral é pequeno ou os dados esparsos, tais métodos podem eventualmente produzir resultados imprecisos. Neste trabalho, revisamos medidas de evidência clássicas e bayesianas comumente empregadas para comparar duas proporções marginais. Além disso, desenvolvemos o Full Bayesian Significance Test (FBST) para testar a homogeneidade marginal em tabelas de contingência bidimensionais e multidimensionais. O FBST é baseado em uma medida de evidência, denominada e-valor, que não depende de resultados assintóticos, não viola o princípio da verossimilhança e respeita a várias propriedades lógicas esperadas para testes de hipóteses. Consequentemente, a abordagem ao problema de teste de homogeneidade marginal pelo FBST soluciona diversas limitações geralmente enfrentadas por outros procedimentos.

Palavras-chave: Full Bayesian Significance Test (FBST), Tabelas de contingência, Teste de homogeneidade marginal. 


\section{Abstract}

CARVALHO, H.G. Bayesian tests for marginal homogeneity in contingency tables. 2015. 65 f. MSc thesis - Instituto de Matemática e Estatística, Universidade de São Paulo, São Paulo, 2015.

Tests of hypotheses for marginal proportions in contingency tables play a fundamental role, for instance, in the investigation of behaviour (or opinion) change. However, most texts in the literature are concerned with tests that assume independent populations (e.g: homogeneity tests). There are some works that explore hypotheses tests for dependent proportions such as the McNemar Test for $2 \times 2$ contingency tables. The generalization of McNemar test for $k \times k$ contingency tables, called marginal homogeneity test, usually requires asymptotic approximations. Nevertheless, for small sample sizes or sparse tables, such methods may occasionally produce imprecise results. In this work, we review some classical and Bayesian measures of evidence commonly applied to compare two marginal proportions. We propose the Full Bayesian Significance Test (FBST) to investigate marginal homogeneity in two-way and multidimensional contingency tables. The FBST is based on a measure of evidence, called e-value, which does not depend on asymptotic results, does not violate the likelihood principle and satisfies logical properties that are expected from hypothesis testing. Consequently, the FBST approach to test marginal homogeneity overcomes several limitations usually met by other procedures.

Keywords: Contingency tables, Full Bayesian Significance Test (FBST), Test of marginal homogeneity. 


\section{Sumário}

Lista de Abreviaturas $\quad$ xi

Lista de Símbolos $\quad$ xiii

Lista de Figuras $\quad$ XV

Lista de Tabelas $\quad$ xvii

1 Introdução $\quad 1$

1.1 Considerações Preliminares . . . . . . . . . . . . . . . . . . . . 1

1.2 Conceitos Básicos . . . . . . . . . . . . . . . . . . . . 2

1.2 .1 Abordagem Clássica . . . . . . . . . . . . . . . . . . 2

1.2 .2 Abordagem Bayesiana . . . . . . . . . . . . . . . . . . . 3

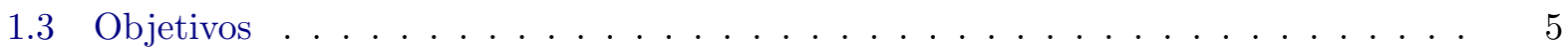

1.4 Organização do Trabalho . . . . . . . . . . . . . . . . . . . 5

$\begin{array}{lll}2 & \text { Testes de hipóteses para proporções pareadas } & 7\end{array}$

2.1 Introdução . . . . . . . . . . . . . . . . . . . . . . . . . 7

2.2 Motivação . . . . . . . . . . . . . . . . . . . . . . . . 7

2.3 Abordagem clássica . . . . . . . . . . . . . . . . . . . . . . 9 9

2.4 Abordagem bayesiana . . . . . . . . . . . . . . . . . . . . 10

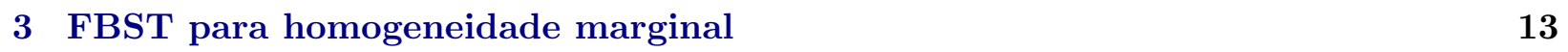

3.1 Introdução . . . . . . . . . . . . . . . . . . . . . . . . . . . . 13

3.2 Motivação . . . . . . . . . . . . . . . . . . . . . . . . 13

3.3 Teste de Homogeneidade Marginal em Tabelas Bidimensionais . . . . . . . . . . . . 14

3.4 Formulação do FBST . . . . . . . . . . . . . . . . . . . . . . . . . 15

3.5 Relações com a hipótese de simetria . . . . . . . . . . . . . . . . . . 17

3.6 Abordagem clássica ao teste de homogeneidade marginal . . . . . . . . . . . . . . 18

3.7 O Modelo Logístico-Normal . . . . . . . . . . . . . . . . . . . . . . . . 20

3.8 Relação entre e-valor e p-valor . . . . . . . . . . . . . . . . . . 24

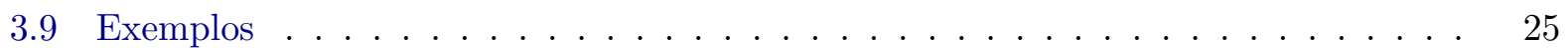

4 Tabelas multidimensionais $\quad \mathbf{2 7}$

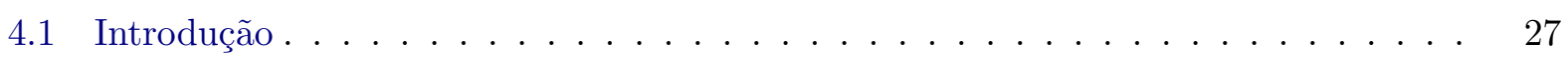

4.2 Motivação . . . . . . . . . . . . . . . . . . . . . . . . . 27 
4.3 Homogeneidade Marginal em Tabelas Tridimensionais . . . . . . . . . . . . . 27

4.4 Hipóteses Lineares . . . . . . . . . . . . . . . . . . . . . . . . . . . 29

5 Conclusões $\quad 31$

5.1 Considerações Finais . . . . . . . . . . . . . . . . . . . . . . 31

5.2 Sugestões para Pesquisas Futuras . . . . . . . . . . . . . . . . 32

A Derivação do p-valor para o teste de McNemar $\quad 33$

B Rotinas Computacionais $\quad \mathbf{3 7}$

B FBST para Homogeneidade Marginal e Simetria em Tabelas Bidimensionais . . . . 37

B FBST para Homogeneidade Marginal em Tabelas Tridimensionais . . . . . . . . . . 40

$\begin{array}{ll}\text { Referências Bibliográficas } & 43\end{array}$ 


\section{Lista de Abreviaturas}

FBST Teste de significância genuinamente bayesiano (Full Bayesian Significance Test)

HPD Máxima densidade a posteriori (Highest Posterior Density)

UMPU Não-viesado uniformemente mais poderoso (UMPU) (Uniformly Most Powerful Unbiased) 


\section{Lista de Símbolos}

$K(p, q)$ Medida de Divergência de Kullback-Leibler das medidas $p$ e $q$. $R V \quad$ Razão de verossimilhanças.

$V_{x}(\theta) \quad$ Função de verossimilhança gerada por $x$ no ponto $\theta$.

$\mathcal{P} \quad$ Conjunto de medidas de probabilidade indexadas por $\theta$.

$\chi \quad$ Espaço amostral.

$\Theta \quad$ Espaço paramétrico.

$\operatorname{logit}(\theta)$ Transformação logito no ponto $\theta$. 


\section{Lista de Figuras}

3.1 Evidências de homogeneidade marginal para os modelos Dirichlet e Logístico-Normal 22

3.2 Medidas de Kullback-Leibler para as tabelas $2 \times 2$ geradas . . . . . . . . . . . . 23

3.3 Evidências de homogeneidade marginal para os modelos Dirichlet e Logístico-Normal 24

3.4 Medida de divergência de Kullback-Leibler para as tabelas 3 x 3 geradas . . . . . . 24

3.5 Relação entre e-valor e p-valor (teste de homogeneidade marginal em tabelas $3 \times 3$ ) 25 


\section{Lista de Tabelas}

1.1 Função de perda $0-a_{1}-a_{2} \ldots \ldots \ldots \ldots$

2.1 Dados de intenção de voto de eleitores em junho e agosto (Agresti, 2013) . . . . 8

3.1 Frequências de acuidade visual dos olhos direito e esquerdo de 7477 funcionários

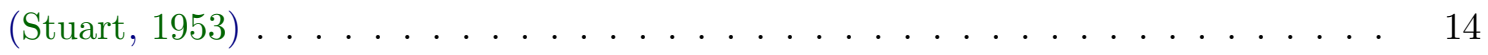

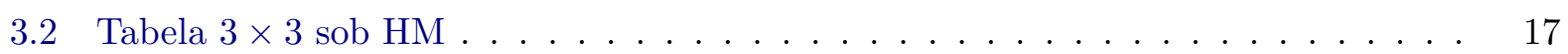

3.3 Número total de tabelas $k \times k$ para um tamanho amostral $n \ldots \ldots \ldots$. . . . . 19

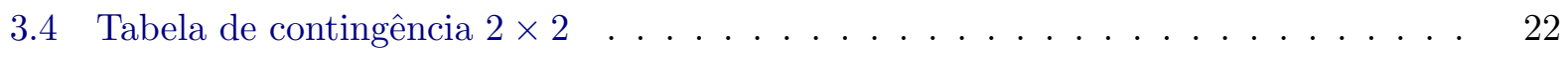

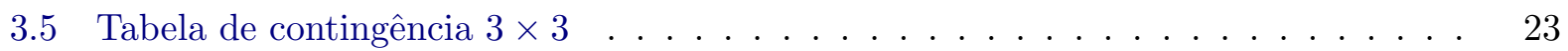

3.6 Dados de mobilidade religiosa (Breen e Hayes, 1997) . . . . . . . . . . . . 26

3.7 Padrões de consumo de café em dois períodos de compra (Agresti, 2007) . . . . . 26

4.1 Dados de intenção de voto ao longo de Junho, Julho e Agosto (Anderson, 1951) . . 28

4.2 Evidência de Pereira-Stern para dados do Exemplo $4.1 \ldots$. . . . . . . . . . . . . . . 29

4.3 Respostas aos tratamentos $A, B$ e $C$ (Grizzle et al., 1969) . . . . . . . . . . 30 


\section{Capítulo 1}

\section{Introdução}

\subsection{Considerações Preliminares}

Em Inferência Estatística, é comum investigar a igualdade de duas proporções assumindo que as populações são independentes. Apesar disso, existem diversas situações em que proporções não podem ser consideradas independentes. Por exemplo, ao conduzir um experimento em que os mesmos indivíduos são questionados sobre sua intenção de voto antes e após um debate presidencial. Neste caso, não seria razoável esperar que as respostas de um indivíduo sejam independentes, o que inviabiliza a análise dos dados por meio de um teste de homogeneidade de proporções (Agresti, 2013).

Os testes de hipóteses para proporções dependentes constituem um problema fundamental em diversas áreas do conhecimento, tais como no estudo de mudança de opinião e comportamento (Irony et al., 2000). Sob a abordagem clássica, McNemar (1947) propôs uma estatística de teste que considera a correlação entre as proporções amostrais. Na literatura, há várias referências sobre esse teste e suas variações, como em Agresti (2013); Holland (2007); Sidik (2001). Nesses testes, o p-valor é a medida de evidência comumente empregada para avaliar a significância estatística da mudança de opinião. No trabalho de McNemar, as variáveis qualitativas assumem dois possíveis atributos, restringindo sua aplicação a apenas tais situações.

A extensão do teste de McNemar para comparações de $k(k>2)$ proporções marginais denomina-se teste de homogeneidade marginal. As soluções clássicas desse problema usualmente requerem o uso de métodos assintóticos ou de reamostragem. Entretanto, quando o tamanho da amostra é pequeno ou os dados são esparsos, os métodos assintóticos podem produzir resultados imprecisos (Hirji, 2005). Embora exista a versão exata do teste de McNemar para tabelas $2 \times 2$, o cálculo do p-valor "exato" em dimensões maiores usualmente requer o uso de técnicas computacionais intensivas. Além disso, o p-valor geralmente exibe inconsistências, como a ausência de coerência (Fossaluza, 2008; Schervish, 1999) e a violação do princípio da verossimilhança (Berger e Wolpert , 1988). Consequentemente, surge a necessidade da definição de uma medida de evidência coerente, que não dependa de resultados assintóticos, não viole o princípio da verossimilhança e seja simples de calcular computacionalmente. O Full Bayesian Significance Test (FBST), proposto por Pereira e Stern (1999), foi idealizado com o objetivo de contornar essas e algumas outras limitações que são geralmente enfrentadas por outras formulações. Ademais, o paradigma bayesiano para testes em tabelas de contingência tem a vantagem de ser apropriado mesmo quando a maioria das contagens são razoavelmente baixas (Good, 1976). 
Deste modo, após examinar os principais testes de hipóteses clássicos e bayesianos para proporções dependentes, adotamos e desenvolvemos o FBST para testar a hipótese de homogeneidade marginal em tabelas de contingência bidimensionais e multidimensionais.

\subsection{Conceitos Básicos}

As medidas de evidência estatística são as mais difundidas no meio científico visando testar hipóteses sobre quantidades desconhecidas. Nesta seção, descrevemos um breve resumo das principais medidas de evidência clássicas e bayesianas utilizadas em testes de hipóteses. Tais medidas serão aplicadas, doravante, em testes de homogeneidade marginal.

Ao longo do trabalho, denotamos por $\Theta$ o espaço paramétrico cujos elementos são representados, genericamente, por $\theta ; \Theta_{0}$ denota o subconjunto do espaço paramétrico que caracteriza a hipótese nula, $\mathcal{X}$ o espaço amostral e $x(\boldsymbol{x})$ o valor (vetor) observado em um experimento. Além disso, $\mathcal{P}=\left\{P_{\theta}: \theta \in \Theta\right\}$ representa uma família de medidas de probabilidade indexadas por $\theta$. Vale notar que, no contexto clássico, não acomoda-se probabilidades em $\Theta$. Neste trabalho, fixamos $\mathcal{P}$ como a família de distribuições Mutinomiais já que esse modelo é adequado quando apenas o tamanho amostral foi previamente fixado (Agresti, 2013). Representamos por $V_{x}(\cdot): \Theta \rightarrow \mathbb{R}_{+}$a função de verossimilhança gerada pela observação $x \mathbb{e}_{A}(\cdot)$ a função indicadora (ou característica) do conjunto $A$ que vale 1 quando $x \in A$ e vale 0 , caso contrário. Finalmente, $\mathcal{F}_{A}$ denota uma $\sigma$-álgebra conveniente de subconjuntos de $A(A \neq \emptyset)$

De maneira geral, uma hipótese estatística é uma afirmação sobre um parâmetro populacional advindo de um modelo estatístico proposto. Um teste para a hipótese $H: \theta \in \Theta_{0}$ contra $A: \theta \in \Theta_{1}$, onde $\Theta_{0} \cap \Theta_{1}=\emptyset$ e $\Theta_{0} \cup \Theta_{1}=\Theta$, é uma função mensurável $\phi$ que associa, para cada $x \in \mathcal{X}, \phi(x)=0$ no caso de decisão por não rejeição de $H$ e $\phi(x)=1$ no caso de decisão por rejeitar $H$. Na maioria dos testes abordados neste trabalho, consideramos que $\Theta_{0}$ tem medida de Lesbesgue nula. Tal hipótese é denominada precisa e sua consideração depende do estatístico possuir motivos para suportar hipóteses deste tipo, seja por se tratar de uma aproximação de hipóteses compostas, seja por razões advindas de fenômenos físicos ou pelo fato de serem comumente abordadas na literatura (Berger e Delampady, 1987; DeGroot, 1970).

\subsubsection{Abordagem Clássica}

Sob a perspectiva clássica, suponha o modelo estatístico $\left(\mathcal{X}, \mathcal{F}_{\mathcal{X}}, \mathcal{P}\right)$. Neste contexto, consideramos a abordagem de Fisher para testes de hipóteses baseada no p-valor. Apesar de existirem diversas definições de p-valor na literatura, adotamos a de Casella e Berger (2002).

Definição 1.2.1 Assuma que existe uma estatística $T: \mathcal{X} \rightarrow \mathbb{R}$ de modo que quanto maior seu valor, menor é a evidência de que a hipótese $H$ descreva o comportamento da amostra observada. $O$ p-valor para testar $H: \theta \in \Theta_{0}$ é definido, para cada $x \in \mathcal{X}$, por

$$
p(x)=\sup _{\theta \in \Theta_{0}} P_{\theta}(T(X)>T(x))
$$


Deste modo, o p-valor é uma medida que avalia a probabilidade de ocorrer um valor da estatística $T$ igualmente ou mais extremo do que o valor efetivamente observado, supondo que a hipótese seja verdadeira. Observe que o p-valor depende da estatística $T$ adotada. Além disso, como o teste da razão de verossimilhanças generalizada é comumente empregado por possuir propriedades desejáveis (como, por exemplo, invariância), restringimos a definição 1.2.1 a esta classe de estatísticas através da definição abaixo.

Definição 1.2.2 Considere o problema de testar a hipótese $H: \theta \in \Theta_{0}$. O p-valor baseado na estatística da razão de verossimilhanças é, para cada $x \in \mathcal{X}$,

$$
p(x)=\sup _{\theta \in \Theta_{0}} P_{\theta}\left(X \in A_{x}\right)
$$

em que $A_{x}=\{z \in \mathcal{X}: R V(z) \leq R V(x)\}$ e $R V(x)=\frac{\sup _{\theta \in \Theta_{0}} V_{x}(\theta)}{\sup _{\theta \in \Theta} V_{x}(\theta)}$ é a estatística da razão de verossimilhanças no ponto $x$.

Desta forma, obtém-se, por meio da ordenação do espaço amostral de acordo com a razão de verossimilhanças, os pontos mais discrepantes à hipótese nula do que o ponto $x$ observado. A obtenção do conjunto $A_{x}$ pode não ser trivial analiticamente, o que pode levar à utilização de métodos de simulação em muitos casos.

\subsubsection{Abordagem Bayesiana}

Assuma um modelo paramétrico bayesiano $\left(\Theta \times \mathcal{X}, \mathcal{F}_{\Theta \times \mathcal{X}}, P\right)$. Sob a perspectiva da teoria da decisão bayesiana, um teste de hipóteses é visto como um problema de decisão em que o espaço de ações, $\mathcal{D}$, possui dois elementos, isto é, $\mathcal{D}=\left\{d_{0}, d_{1}\right\}$, onde $d_{0}$ é a decisão de aceitar a hipótese nula $H$ e $d_{1}$ a decisão de aceitar a hipótese alternativa $A$. Assim, associa-se uma função $L: \mathcal{D} \times \Theta \rightarrow \mathbb{R}_{+}$ que especifica, para cada par $(d, \theta)$, a perda incorrida ao tomar a decisão $d$ quando o estado da natureza é $\theta \in \Theta$. Para testar as hipóteses $H: \theta \in \Theta_{0}$ contra $A: \theta \in \Theta_{1}$, atribui-se perdas as possíveis decisões quando o estado da natureza é $\Theta_{0}$ ou $\Theta_{1}$. Usualmente, adota-se a função de perda da Tabela 1.1, denominada perda $0-a_{1}-a_{2}$ (Schervish, 1995). Daí, uma maneira de testar hipóteses precisas da forma $H: \theta=\theta_{0}$ contra $A: \theta \neq \theta_{0}, \theta_{0} \in \Theta$, consiste em atribuir uma probabilidade positiva a priori $\delta$ para $H$, isto é, $P\left(\theta=\theta_{0}\right)=\delta>0$, como desenvolvido por Jeffreys (1961).

Tabela 1.1: Função de perda $0-a_{1}-a_{2}$

\begin{tabular}{rrr}
\hline & $\theta \in \Theta_{0}$ & $\theta \in \Theta_{1}$ \\
\hline$d_{0}$ & 0 & $a_{1}$ \\
$d_{1}$ & $a_{2}$ & 0 \\
\hline
\end{tabular}

Deste modo, a probabilidade a posteriori de $H$ dado $x$ é

$$
P\left(\theta=\theta_{0} \mid X=x\right)=\frac{\delta f\left(x \mid \theta_{0}\right)}{\delta f\left(x \mid \theta_{0}\right)+(1-\delta) \int_{\Theta} f(x \mid \theta) g(\theta) d \theta}
$$


em que $g(\cdot)$ é a função densidade de probabilidade a priori sobre $\Theta$. Utilizando a função de perda $0-a_{1}-a_{2}$, temos que o teste de Bayes rejeita $H$ se, e somente se,

$$
P\left(\theta=\theta_{0} \mid X=x\right)<\frac{a_{1}}{a_{1}+a_{2}} .
$$

O FBST (Pereira e Stern, 1999) foi desenvolvido como uma alternativa genuinamente bayesiana para contornar algumas dificuldades geralmente enfrentadas por testes clássicos e bayesianos. Por exemplo, o FBST não requer a introdução de uma probabilidade a priori positiva para o subconjunto definido pela hipótese precisa, diferentemente dos testes de Jeffreys. O FBST é baseado em uma medida de evidência, denominada e-valor, que descrevemos na sequência.

Definição 1.2.3 Suponha que, a priori, $\theta$ seja distribuído segundo uma medida de probabilidade absolutamente contínua em relação à medida Lebesgue cuja função densidade de probabilidade a priori é $f(\cdot)$. Suponha que, para todo $x \in \mathcal{X}, f(\cdot \mid x)$ é a função densidade de probabilidade da distribuição a posteriori $\theta \mid x$. Para cada $x \in \mathcal{X}$, seja $T_{x}=\left\{\theta \in \Theta: f(\theta \mid x)>\sup _{\theta \in \Theta_{0}} f(\theta \mid x)\right\}$ o conjunto tangente a $\Theta_{0}$, sendo $\Theta_{0}$ o subconjunto de $\Theta$ caracterizado pela hipótese $H$. O e-valor em favor de $H$ é definido, para cada $x \in \mathcal{X}$, por

$$
e v\left(\Theta_{0} ; x\right)=1-P\left(\theta \in T_{x} \mid x\right)
$$

A região tangente consiste no conjunto HPD (Highest Posterior Density) de pontos que possuem densidade a posteriori maior do que qualquer elemento de $\Theta_{0}$. O FBST é um procedimento que rejeita $H$ quando ev $\left(\Theta_{0} ; x\right)$ é pequena. Vale ressaltar que a densidade a posteriori é o único elemento necessário para o cálculo do e-valor. Note ainda que o FBST não requer qualquer procedimento de redução da dimensionalidade do espaço paramétrico devido a eventual presença de parâmetros de perturbação (Pereira et al., 2008). Adicionalmente, Madruga et al. (2001) mostram que o FBST é um teste de Bayes, pois, sob certas condições, existe uma função de perda que torna o FBST um procedimento que minimiza a correspondente perda esperada.

A formulação inicial do FBST descrita acima não respeita a propriedade de invariância sobre transformações bijetivas no espaço paramétrico original (Madruga et al., 2002). Com isso, a evidência para testar $H: \theta \in \Theta_{0}$ em geral não coincide com a evidência para testar $H^{\prime}$ : $\phi(\theta) \in \phi\left(\Theta_{0}\right)$, sendo $\phi$ uma função bijetora, mensurável e integrável. Para contornar essa limitação, Madruga et al. (2002) introduzem uma densidade referência, $r(\theta)$, sobre $\Theta$ e definem

$$
\theta^{*}=\arg \max _{\theta \in \Theta_{0}}\left\{\frac{f(\theta \mid x)}{r(\theta)}\right\} \text { e } s_{x}^{*}=\max _{\theta \in \Theta_{0}}\left\{\frac{f(\theta \mid x)}{r(\theta)}\right\}
$$

A função $s_{x}(\cdot)$ é denominada surpresa relativa (Good, 1956). O conjunto com a maior surpresa relativa $T^{*}$ contém os pontos do espaço paramétrico cuja surpresa relativa é maior do que a máxima surpresa relativa em $\Theta_{0}$, ou seja, 


$$
T_{x}^{*}=\left\{\theta \in \Theta: \frac{f(\theta \mid x)}{r(\theta)}>s_{x}^{*}\right\}
$$

Assim, a evidência em favor de $H$ é redefinida por

$$
e v^{*}\left(\Theta_{0} ; x\right)=1-P\left(\theta \in T_{x}^{*} \mid x\right)
$$

Madruga et al. (2002) mostram que a evidência (1.6) é invariante ao tomar reparametrizações bijetoras. Tal resultado é útil, por exemplo, ao considerar testes de hipóteses para tabelas de contingência nesse trabalho. A evidência 1.6 será empregada na Seção 3.7, ao desenvolvermos o FBST para testar homogeneidade marginal considerando o modelo Logístico-Normal, enquanto que adotaremos a definição 1.5 ao longo das outras seções.

\subsection{Objetivos}

Os objetivos deste trabalho são:

- Estudar a construção dos testes de hipóteses para comparar duas proporções marginais em tabelas de contingência. Neste contexto, revisamos algumas propostas para calcular p-valores e medidas de evidência baseadas na probabilidade a posteriori.

- Propor uma medida de evidência coerente (e-valor) para testes de homogeneidade marginal em tabelas bidimensionais bem como revisar brevemente alguns procedimentos utilizados sob a abordagem clássica.

- Desenvolver o FBST para testes de homogeneidade marginal em tabelas multidimensionais, aplicando tal procedimento em exemplos específicos.

\subsection{Organização do Trabalho}

No Capítulo 2, revisamos as principais medidas de evidência utilizadas para testar a homogeneidade de proporções marginais quando observa-se variáveis que assumem valores em um conjunto com duas possíveis categorias. No Capítulo 3, abordamos o FBST para a hipótese de homogeneidade marginal em uma tabela de contingência $k \times k, k>2$. Estabelecemos a generalização multidimensional do teste de homogeneidade marginal no Capítulo 4. No Capítulo 5, descrevemos as principais conclusões obtidas neste trabalho e apresentamos perspectivas para futuras pesquisas. 


\section{Capítulo 2}

\section{Testes de hipóteses para proporções pareadas}

\subsection{Introdução}

A comparação de duas proporções marginais em uma tabela de contingência $2 \times 2$ é uma prática frequente em diversas áreas do conhecimento. Na literatura, tal comparação foi empregada para avaliar a confiabilidade de dados em ensaios clínicos (Klingenberg e Agresti, 2006), investigar taxas de erros de dois algoritmos de classificação (Hastie et al., 2009), analisar a dinâmica de intenções de voto ao longo de diferentes ocasiões (Agresti, 2013), averiguar a presença de linkage entre um marcador genético e um determinado fenótipo (Ziegler e König, 2010), dentre outras aplicações. A forma comumente utilizada para avaliar a compatibilidade dos dados com a hipótese de homogeneidade de duas proporções marginais é através do p-valor. Neste capítulo, revisamos as principais propostas de p-valor para este teste assim como a formulação bayesiana baseada na probabilidade a posteriori da hipótese a ser testada.

\subsection{Motivação}

Exemplo 2.1. (Agresti, 2013) No período que antecede uma eleição majoritária com dois candidatos, decidiu-se conduzir um experimento para analisar se os eleitores de uma determinada região mudaram sua opinião ao longo da campanha presidencial. Assim, para cada indivíduo da amostra, observa-se um par $\left(a_{r}, b_{r}\right)$ onde $a_{r}$ corresponde à intenção de voto do r-ésimo eleitor no mês de junho e $b_{r}$ sua intenção em uma segunda entrevista, no mês de agosto. O conjunto de possíveis valores de $a_{r}$ e $b_{r}$ é $\mathbb{C}=\{$ Democrata (1), Republicano (2) $\}$.

Usualmente, para fins de inferência, utiliza-se o vetor aleatório $\boldsymbol{Y}=\left(n_{11}, n_{12}, n_{21}, n_{22}\right)$ representado em uma tabela de contingência $2 \times 2$ (Tabela 2.1), em que $n_{i j}$ é o número de indivíduos que tem a intenção de votar no candidato $i$ de $\mathbb{C}$ na pesquisa de Junho e optam pelo candidato $j$ de $\mathbb{C}$ na pesquisa de Agosto. Descrevemos, ao longo desse capítulo, algumas alternativas para investigar a significância estatística da mudança de opinião dos eleitores.

Assuma o modelo multinomial para $\boldsymbol{Y}$, fixado $\boldsymbol{\theta}=\left(\theta_{11}, \theta_{12}, \theta_{21}, \theta_{22}\right)$, em que $\theta_{i j}$ é a probabilidade de um indivíduo pertencer a célula $(i, j), i, j=1,2$. Sob estas suposições, podemos formular a hipótese de que a opinião dos indivíduos não se altera como $H: \theta_{1+}=\theta_{+1}$, onde $\theta_{1+}=\theta_{11}+\theta_{12} \mathrm{e}$ 
Tabela 2.1: Dados de intenção de voto de eleitores em junho e agosto (Agresti, 2013)

\begin{tabular}{cccc}
\hline & \multicolumn{2}{c}{ Agosto } & \\
\cline { 2 - 3 } Junho & 1 & 2 & Total \\
\hline 1 & 142 & 38 & 180 \\
2 & 32 & 429 & 461 \\
Total & 174 & 467 & 641 \\
\hline
\end{tabular}

$\theta_{+1}=\theta_{11}+\theta_{21}$, denominada hipótese de homogeneidade marginal que é, neste cenário, equivalente à condição de simetria $\theta_{12}=\theta_{21}$.

Seguindo as definições do Capítulo 1, estamos testando, sob a abordagem clássica, se $P$ pertence à família $\mathcal{P}_{0}$ que engloba todas as distribuições Multinomiais sujeitas à restrição $\theta_{12}=\theta_{21}$.

Note que podemos considerar apenas os pares discordantes pois, fixado o tamanho amostral, as contagens individuais $n_{11}$ e $n_{22}$ não trazem informação sobre $\theta_{12}$ ou $\theta_{21}$. Assim, considere o vetor $\boldsymbol{X}=\left(n_{12}, n_{21}, n_{11}+n_{22}\right)$, de modo que $\boldsymbol{X} \mid\left(\theta_{12}, \theta_{21}, \theta^{d}\right) \sim \operatorname{Multinomial}\left(n,\left(\theta_{12}, \theta_{21}, \theta^{d}\right)\right)$. Seja $t=n_{12}+n_{21}$, então a função de verossimilhança gerada por $\boldsymbol{x}$ é dada por

$$
V_{\boldsymbol{x}}\left(\theta_{12}, \theta_{21}, \theta^{d}\right)=\left(\begin{array}{c}
n \\
n_{12}, n_{21}
\end{array}\right) \theta_{12}^{n_{12}} \theta_{21}^{n_{21}}\left(\theta^{d}\right)^{n-t} \mathbb{I}_{\left\{(a, b, c) \in \mathbb{R}_{+}^{3}: a+b+c=1\right\}}\left(\theta_{12}, \theta_{21}, \theta^{d}\right)
$$

Sob $H: \theta_{12}=\theta_{21}=\theta$, a verossimilhança fica dada por

$$
V_{\boldsymbol{x}}(\theta)=\left(\begin{array}{c}
n \\
n_{12}, n_{21}
\end{array}\right) \theta^{t}(1-2 \theta)^{n-t} \mathbb{I}_{[0,1 / 2]}(\theta)
$$

A obtenção do p-valor a partir de 2.2 segundo a Definição 1.2 é de difícil tratamento analítico. Contudo, manipulando a Equação 2.1, obtém-se que

$$
V_{\boldsymbol{x}}\left(\theta_{12}, \theta_{21} \theta^{d}\right)=\left(\begin{array}{c}
n \\
t
\end{array}\right)\left(\begin{array}{c}
t \\
n_{12}
\end{array}\right)\left(\frac{\theta_{12}}{\theta_{12}+\theta_{21}}\right)^{n_{12}}\left(\frac{\theta_{21}}{\theta_{12}+\theta_{21}}\right)^{n_{21}}\left(1-\theta^{d}\right)^{t}\left(\theta^{d}\right)^{n-t}
$$

Seja $\phi=\frac{\theta_{12}}{\theta_{12}+\theta_{21}}$ e $\eta=1-\theta^{d}$, então

$$
V_{\boldsymbol{x}}(\eta, \phi)=\left(\begin{array}{c}
t \\
n_{12}
\end{array}\right) \phi^{n_{12}}(1-\phi)^{t-n_{12}}\left(\begin{array}{c}
n \\
t
\end{array}\right) \eta^{t}(1-\eta)^{n-t}
$$

isto é,

$$
V_{\boldsymbol{x}}(\eta, \phi)=V_{n_{12} \mid t}^{\prime}(\phi) V_{t}^{\prime \prime}(\eta)
$$

onde $V_{n_{12} \mid t}^{\prime}(\phi)=\left(\begin{array}{c}t \\ n_{12}\end{array}\right) \phi^{n_{12}}(1-\phi)^{t-n_{12}}$ e $V_{t}^{\prime \prime}(\eta)=\left(\begin{array}{c}n \\ t\end{array}\right) \eta^{t}(1-\eta)^{n-t}$.

Se a função de verossimilhança gerada por $\boldsymbol{x}$ puder ser fatorada em duas componentes, como em 2.3, então dizemos que os parâmetros $\phi$ e $\eta$ são ortogonais (Cox e Reid, 1987). Com isso, existe uma reparametrização $\boldsymbol{\tau}=(\eta, \phi)$ do modelo na qual a verossimilhança de $\boldsymbol{x}$ é fatorada em duas 
componentes ortogonais. A primeira componente, $V_{n_{12} \mid t}^{\prime}(\phi)$ é denominada função de verossimilhança condicional, pois trata-se da função de verossimilhança baseada na distribuição condicional de $n_{12} \mid t, \phi$ (Pace e Salvan, 1997). Já a segunda componente, $V_{t}^{\prime \prime}(\eta)$, é denominada função de verossimilhança marginal, pois trata-se da função de verossimilhança baseada na distribuição de $t \mid \eta$. Em decorrência da ortogonalidade, segue que não há perda de informação ao considerar apenas $V_{n_{12} \mid t}^{\prime}(\phi)$ para estimar $\phi$ (Basu, 1977). Alguns autores consideram a verossimilhança condicional para obter p-valores, denominados de p-valores condicionais (Silvapulle e Sen, 2005). Baseando-se nesse modelo condicional, derivamos, nas próximas seções, o p-valor condicional para testar $H$, bem como as probabilidades a posteriori dessa hipótese.

\subsection{Abordagem clássica}

Nesta seção, consideramos o problema de testar a igualdade das proporções marginais em tabelas de contingência $2 \times 2$, denominado teste exato de McNemar (Agresti, 2013). Exibimos o cálculo do p-valor condicional, seguindo as ideias da seção 2.2 .

Resultado 2.3.1 Suponha um vetor aleatório $\boldsymbol{X}=\left(n_{21}, n_{21}, n_{11}+n_{22}\right)$ que, fixado $\boldsymbol{\theta}=\left(\theta_{12}, \theta_{21}, \theta^{d}\right)$, possui distribuição Multinomial $(n ; \boldsymbol{\theta})$, onde $t=n_{12}+n_{21}$. O p-valor condicional, $p: \mathcal{X}^{\prime} \rightarrow[0,1]$, para testar $H: \theta_{12} \leq \theta_{21}$ contra $A: \theta_{12}>\theta_{21}$ baseado na estatística da razão de verossimilhanças generalizada condicional é, para cada $n_{12} \in \mathcal{X}^{\prime}$,

$$
p\left(n_{12}\right)= \begin{cases}P\left(\operatorname{Bin}\left(t, \frac{1}{2}\right) \geq n_{12}\right), & \text { se } n_{12}>t / 2 \\ 1, & \text { caso contrário }\end{cases}
$$

onde $t=n_{12}+n_{21}$ e $\mathcal{X}^{\prime}=\{0, \ldots, t\}$.

Demonstração: Dada no Apêndice A.

Na sequência, descrevemos um resultado que permite calcular o p-valor condicional do teste exato de McNemar.

Resultado 2.3.2 Suponha um vetor aleatório $\boldsymbol{X}=\left(n_{12}, n_{21}, n_{11}+n_{22}\right)$ que, fixado $\boldsymbol{\theta}$, possui distribuição Multinomial $\left(n ;\left(\theta_{12}, \theta_{21}, \theta^{d}\right)\right)$, onde $t=n_{12}+n_{21}$. O p-valor condicional, $p: \mathcal{X}^{\prime} \rightarrow[0,1]$, para testar $H: \theta_{12}=\theta_{21}$ contra $A: \theta_{12} \neq \theta_{21}$ baseado na estatística da razão de verossimilhanças generalizada condicional é, para cada $n_{12} \in \mathcal{X}^{\prime}$,

$$
p^{*}\left(n_{12}\right)= \begin{cases}2 P\left(\operatorname{Bin}\left(t, \frac{1}{2}\right) \geq n_{12}\right), & \text { se } n_{12} \geq t / 2 \\ 2 P\left(\operatorname{Bin}\left(t, \frac{1}{2}\right) \leq n_{12}\right), & \text { caso contrário }\end{cases}
$$

onde $t=n_{12}+n_{21}$ e $\mathcal{X}^{\prime}=\{0, \ldots, t\}$.

Demonstração: Dada no Apêndice A.

Utilizando os dados da Tabela 2.1 e o Resultado 2.3.1, segue que o p-valor do teste unilateral é 0.275 , indicando que não há evidências para rejeitar que a opinião dos eleitores não se alterou 
ou de que ocorreu um aumento na proporção de votos entre Junho e Agosto para o candidato do partido Democrata. Pelo Resultado A.2, o p-valor do teste bilateral é 0.550, concluindo que não há evidências para rejeitar que a opinião dos eleitores não se alterou entre Junho e Agosto. Embora os p-valores tenham sido ambos derivados da estatística da razão de verossimilhanças, há mais evidências para não rejeitar a hipótese $\theta_{12}=\theta_{21}$ do que para não rejeitar $\theta_{12} \leq \theta_{21}$, ilustrando certa incoerência lógica do p-valor, como discutido em Schervish (1996).

\subsection{Abordagem bayesiana}

A fim de desenvolver o teste de Bayes para homogeneidade marginal em tabelas $2 \times 2$, derivamos abaixo um resultado importante para representar a função de distribuição de uma variável aleatória Beta em termos da cauda superior de uma distribuição Binomial, possibilitando obter comparações dos testes bayesianos a seguir com os resultados obtidos sob a abordagem clássica.

Resultado 2.4.1 Suponha que $\theta \sim \operatorname{Beta}(a, b)$, com $a, b \in \mathbb{N}^{*}$. Seja $f(\cdot \mid a, b)$ a função densidade de $\theta$. Então, para $0<p<1$,

$$
\int_{0}^{p} f(\theta \mid a, b) d \theta=\sum_{j=a}^{a+b-1} \frac{(a+b-1) !}{j !(a+b-j-1) !} p^{j}(1-p)^{a+b-j-1} .
$$

Demonstração: Utilizando integração por partes, temos que

$$
\begin{aligned}
\int_{0}^{p} \frac{1}{B(a, b)} \theta^{a-1}(1-\theta)^{b-1} d \theta & =\left.\frac{(a+b-1) !}{(a)(a-1) !(b-1) !}(1-\theta)^{b-1} \theta^{a}\right|_{0} ^{p} \\
+\int_{0}^{p} \frac{\theta^{a}(1-\theta)^{b-2}}{a} \frac{(a+b-1) !}{(a-1) !(b-2) !} d \theta & =\frac{(a+b-1) !}{a !(b-1) !} p^{a}(1-p)^{b-1}+ \\
+\int_{0}^{p} f\left(\theta \mid a^{\prime}, b^{\prime}\right) d z &
\end{aligned}
$$

onde $a^{\prime}=a+1$ e $b^{\prime}=b-1$. Aplicando essa expressão iterativamente, segue que

$$
\int_{0}^{p} f(\theta \mid a, b) d \theta=\sum_{j=a}^{a+b-1} \frac{(a+b-1) !}{j !(a+b-j-1) !} p^{j}(1-p)^{a+b-j-1}
$$

Sob a abordagem bayesiana, consideramos $\boldsymbol{X} \mid \boldsymbol{\theta} \sim \operatorname{Multinomial}(n, \boldsymbol{\theta})$ e $\boldsymbol{\theta} \sim \operatorname{Dirichlet}(\boldsymbol{\alpha})$, o que, pelo teorema de Bayes, resulta em $\boldsymbol{\theta} \mid \boldsymbol{x} \sim \operatorname{Dirichlet}(\boldsymbol{\alpha}+\boldsymbol{x})$.

Resultado 2.4.2 O teste de Bayes para as hipóteses $H: \theta_{12} \leq \theta_{21}$ contra $A: \theta_{12}>\theta_{21}$, com relação a perda $0-1$ rejeita $H$ se, e somente se, $P(H \mid x)<\frac{1}{2}$, isto é,

$$
P(H \mid x)=P\left(\operatorname{Beta}\left(v_{12}, v_{21}\right) \leq \frac{1}{2}\right)
$$


onde $v_{12}=n_{12}+\alpha_{12}, v_{21}=n_{21}+\alpha_{21}$. Em particular, se $\alpha_{12}, \alpha_{21} \in \mathbb{N}$,

$$
P(H \mid \boldsymbol{x})=\sum_{j=0}^{v_{12}-1}\left(\begin{array}{c}
v_{12}+v_{21}-1 \\
j
\end{array}\right)\left(\frac{1}{2}\right)^{v_{12}+v_{21}-1} .
$$

Demonstração: Observe que

$$
P\left(\theta_{12} \leq \theta_{21} \mid \boldsymbol{x}\right)=P\left(\frac{\theta_{12}}{\theta_{12}+\theta_{21}} \leq \frac{1}{2} \mid \boldsymbol{x}\right)=P\left(\phi \leq \frac{1}{2} \mid \boldsymbol{x}\right)
$$

Sob esta reparametrização, testamos se $H: \phi \leq \frac{1}{2}$ contra $A: \phi>\frac{1}{2}$. Pela caracterização da distribuição Dirichlet por razão de Gamas, é possível mostrar que $\phi \mid \boldsymbol{x} \sim \operatorname{Beta}\left(v_{12}, v_{21}\right)$ (Irony et al., 2000). Logo, a probabilidade a posteriori da hipótese $H$ é

$$
P\left(\phi \leq \frac{1}{2} \mid \boldsymbol{x}\right)=P\left(\operatorname{Beta}\left(v_{12}, v_{21}\right) \leq \frac{1}{2}\right)
$$

Especificamente, se $\alpha_{12}, \alpha_{21} \in \mathbb{N}$, temos, aplicando o Resultado 2.4.1, que

$$
P(H \mid \boldsymbol{x})=\sum_{j=v_{12}}^{v_{12}+v_{21}-1}\left(\begin{array}{c}
v_{12}+v_{21}-1 \\
j
\end{array}\right)\left(\frac{1}{2}\right)^{v_{12}+v_{21}-1} .
$$

Tomando $\alpha_{21}=1$ e $\alpha_{12}=1 / m, m>0$,

$$
\lim _{m \rightarrow \infty} P\left(\phi \leq \frac{1}{2} \mid \boldsymbol{x}\right)=p(\boldsymbol{x})
$$

Consequentemente, o p-valor não coincide com a probabilidade a posteriori da hipótese $H$ ao considerar uma distribuição a priori Beta, a menos que tomemos sua forma limite.

Derivamos, a seguir, a probabilidade a posteriori de $\phi=1 / 2$ para testar a hipótese bilateral de homogeneidade marginal em tabelas $2 \times 2$, admitindo uma probabilidade positiva a priori para $H$, como em Jeffreys (1961).

Resultado 2.4.3 O procedimento de Bayes para testar $H: \theta_{12}=\theta_{21}$ contra $A: \theta_{12} \neq \theta_{21}$, com relação a perda $0-1$, rejeita $H$ se, e somente se, $P^{\prime}(\phi=1 / 2 \mid \boldsymbol{x})<\frac{1}{2}$, isto é, se

$$
P^{\prime}(\phi=1 / 2 \mid \boldsymbol{x})=\left[1+\frac{1-\delta}{\delta} \frac{B\left(\alpha_{12}, \alpha_{21}\right)}{2^{n_{12}+n_{21}} B\left(v_{12}, v_{21}\right)}\right]^{-1}<1 / 2
$$

sendo $\delta=P^{\prime}(H)>0$ e $v_{12}, v_{21}, \alpha_{12}, \alpha_{21}$ como no Resultado 2.4.2.

Demonstração: Considerando o modelo condicional, o teste é reescrito como $H: \phi=\frac{1}{2}$ contra $A: \phi \neq \frac{1}{2}$. Considerando a priori uma probabilidade positiva para $H, P^{\prime}(\phi=1 / 2)=\delta>0$, 
segue que $g$ na representação 1.3 corresponde à densidade $\operatorname{Beta}\left(\alpha_{12}, \alpha_{21}\right)$. Logo, a probabilidade a posteriori de $H: \phi=\frac{1}{2}$ dado $x$ é

$$
\begin{aligned}
P^{\prime}(\phi=1 / 2 \mid \boldsymbol{x}) & =\frac{\delta p\left(n_{12} \mid \phi=\frac{1}{2}\right)}{\delta p\left(n_{12} \mid \phi=\frac{1}{2}\right)+(1-\delta) \int_{0}^{1} p\left(n_{12} \mid \phi\right) g(\phi) d \phi}=\frac{\delta \frac{1}{2^{n_{12}+n_{21}}}}{\delta \frac{1}{2^{n_{12}+n_{21}}}+(1-\delta) B\left(v_{12} ; v_{21}\right)}= \\
& =\left[1+\frac{1-\delta}{\delta} 2^{n_{12}+n_{21}} B\left(v_{12}, v_{21}\right)\right]^{-1} \cdot
\end{aligned}
$$

Considerando a perda $0-1$, rejeita-se $H$ quando $P(\phi=1 / 2 \mid \boldsymbol{x})<\frac{1}{2}$, daí segue o resultado.

Aplicando o Resultado 2.4.2 aos dados da Tabela considerando a priori uma distribuição Beta $(1,1)$, temos $P(\phi \leq 1 / 2 \mid \boldsymbol{x})=0,24$. Do mesmo modo, aplicando o Resultado 2.4 .3 considerando $g$ a densidade $\operatorname{Beta}(1,1)$, temos $P^{\prime}(\phi=1 / 2 \mid \boldsymbol{x})=0,84$. Observe que há certa artificialidade gerada pela atribuição de uma medida positiva no ponto $1 / 2$. Sob a noção subjetiva de probabilidade, não é razoável utilizar duas medidas de probabilidade a priori distintas para representar a opinião de um indivíduo. Assim, no próximo capítulo, buscamos definir uma medida de evidência geral que não dependa da atribuição de uma probabilidade positiva para hipóteses precisas e, adicionalmente, respeite algumas propriedades lógicas esperadas pelos testes de hipóteses. 


\section{Capítulo 3}

\section{FBST para homogeneidade marginal}

\subsection{Introdução}

Existem diversas situações em que busca-se investigar a igualdade das proporções marginais de duas variáveis qualitativas que assumem valores no mesmo conjunto. Essa avaliação é frequentemente conduzida, por exemplo, quando:

- um grupo de indivíduos é classificado de acordo com duas variáveis categóricas que são dependentes (ex.: nível da qualidade da visão nos olhos direito e esquerdo);

- cada indivíduo é acompanhado em dois pontos do tempo e classificado segundo a mesma estrutura de categorização (ex.: análise da dinâmica de intenções de votos antes e depois do debate que antecede uma eleição majoritária);

- observa-se, na amostra, indivíduos que foram pareados no experimento (ex.: estudos genéticos sobre os padrões de transmissão de um alelo dos pais para sua prole).

O teste de McNemar, descrito anteriormente, só pode ser aplicado quando há duas categorias possíveis de resposta, tornando-se inadequado em problemas com múltiplas categorias. Neste capítulo, descrevemos a ideia geral subjacente à homogeneidade marginal em tabelas de contingência, $k \times k, k>2$, bem como a construção de testes para esta hipótese considerando o paradigma bayesiano, mais especificamente, sob a abordagem do FBST (Full Bayesian Significance Test). Ao longo deste capítulo, estamos supondo que as variáveis discretas são nominais, isto é, não há natureza de ordem entre seus possíveis valores. Notamos também que poderíamos considerar diversos modelos estatísticos em função da regra de parada do experimento, contudo tratamos apenas do modelo Multinomial, isto é, assumindo que o tamanho amostral foi previamente fixado. Estabelecemos também comparações com testes de significância clássicos baseados na estatística de Stuart-Maxwell na Seção 3.6. Além disso, aproximações da posteriori através do modelo Logístico-Normal são comentadas na Seção 3.7. Na seção 3.8, exploramos, descritivamente, a relação entre o p-valor e o e-valor.

\subsection{Motivação}

Exemplo 3.1. (Stuart, 1953) A Tabela 3.1 apresenta registros dos testes de acuidade visual de 7477 funcionários com idades de 30-39 anos das indústrias de artilharia da Inglaterra durante o final 
da $2^{\text {a }}$ guerra mundial (Stuart, 1953). Considere $\mathbb{C}=\{$ Alto (1), Moderado (2), Baixo (3), Muito baixo (4) $\}$ o conjunto de possíveis graus de acuidade visual. Seja $\theta_{i j}$ a probabilidade da visão do olho direito do indivíduo ser classificado na j-ésima categoria de $\mathbb{C}$ e a do olho esquerdo na i-ésima categoria, $i, j=1,2,3,4$. O espaço paramétrico é $\Theta=\left\{\boldsymbol{\theta}=\left(\theta_{11}, \theta_{12}, \theta_{13}, \theta_{14}, \ldots, \theta_{41}, \theta_{42}, \ldots, \theta_{44}\right) \in\right.$ $\left.\mathbb{R}_{+}^{16}, \sum_{i=1}^{4} \sum_{j=1}^{4} \theta_{i j}=1\right\}$. Assuma que $\left(n_{i j}\right)_{i, j=1,2,3,4} \mid \boldsymbol{\theta} \sim \operatorname{Multinomial}(n, \boldsymbol{\theta})$. O espaço amostral é $\mathcal{X}=\left\{\left(n_{11}, n_{12}, \ldots, n_{14}, \ldots, n_{41}, \ldots, n_{44}\right) \in \mathbb{N}^{16}: \sum_{i=1}^{4} \sum_{j=1}^{4} n_{i j}=7477\right\}$, onde $n_{i j}$ representa a contagem da célula $(i, j), i, j=1, \ldots, 4$.

Tabela 3.1: Frequências de acuidade visual dos olhos direito e esquerdo de 7477 funcionários (Stuart, 1953)

\begin{tabular}{cccccc}
\hline & \multicolumn{5}{c}{ Olho direito } \\
Olho esquerdo & 1 & 2 & 3 & 4 & Total \\
1 & 1520 & 266 & 124 & 66 & $1976(0,264)$ \\
2 & 234 & 1512 & 432 & 78 & $2256(0,302)$ \\
3 & 117 & 362 & 1772 & 205 & $2456(0,328)$ \\
4 & 36 & 82 & 179 & 492 & $789(0,106)$ \\
Total & $1907(0,256)$ & $2222(0,297)$ & $2507(0,335)$ & $841(0,112)$ & 7477 \\
\hline
\end{tabular}

Neste cenário, uma hipótese de interesse é dada por: $H^{M}: \theta_{i+}=\theta_{+i}, i=1,2,3$, em que $\theta_{i+}=$ $\sum_{j=1}^{4} \theta_{i j}$ e $\theta_{+i}=\sum_{j=1}^{4} \theta_{j i}$, ou seja, $H^{M}$ significa que as acuidades visuais dos dois olhos são identicamente distribuídas (homogeneidade marginal). Uma outra hipótese que pode ser investigada é a de simetria $H^{S}: \theta_{i j}=\theta_{j i}, i, j \in \mathbb{C}, i \neq j$. Através do teste de Stuart-Maxwell para $H^{M}$ (Stuart , 1953), obtemos um p-valor igual a 0,009, levando a conclusão de que há fortes indícios para rejeitar tal hipótese. Baseando-se na generalização da estatística de McNemar (Bowker, 1948), o p-valor para testar $H^{S}$ é 0,080 , não indicando forte evidência para rejeitá-la. Com isso, adotando um nível de significância de 5\% (ou de 1\%), concluímos que as distribuições das qualidades das visões são diferentes, enquanto que não rejeitamos que a distribuição conjunta é simétrica, o que parece contraditório pois $H^{S} \subset H^{M}$ (Agresti, 2013). A fim de contornar as inconsistências lógicas advindas do p-valor (como a ilustrada acima), do fator de Bayes e outras medidas de evidência, desenvolvemos, ao longo deste capítulo, o FBST para essas hipóteses. O FBST baseia-se numa medida de evidência genuinamente bayesiana que é coerente no sentido de Schervish (1995).

\subsection{Teste de Homogeneidade Marginal em Tabelas Bidimensio- nais}

Suponha um vetor aleatório $\boldsymbol{X}$ distribuído, condicionalmente a $\boldsymbol{\theta}$, segundo o modelo $\operatorname{Multinomial}(n, \boldsymbol{\theta})$, onde $n$ é o tamanho amostral previamente fixado e $\Theta=\left\{\boldsymbol{\theta}=\left(\theta_{11}, \theta_{12}, \ldots, \theta_{1 k}, \theta_{21}, \theta_{22}, \ldots, \theta_{2 k}, \ldots, \theta_{k 1}, \theta_{k 2}\right.\right.$, $\left.\left.\ldots, \theta_{k k}\right) \in \mathbb{R}_{+}^{k \times k}, \sum_{i=1}^{k} \sum_{j=1}^{k} \theta_{i j}=1\right\}$. Considere ainda que o espaço amostral é $\mathcal{X}=\left\{\left(n_{11}, n_{12}, \ldots, n_{1 k}, n_{21}, n_{22}, \ldots, n_{2 k}, \ldots, n_{k 1}, n_{k 2}, \ldots, n_{k k}\right) \in \mathbb{N}^{k \times k}: n_{++}=\sum_{i=1}^{k} \sum_{j=1}^{k} n_{i j}=\right.$ $n\}$. De forma análoga ao Exemplo 3.1, testar a homogeneidade marginal consiste em verificar a hipótese de igualdade das proporções marginais, isto é,

$$
H^{M}: \theta_{i+}=\theta_{+i}, i=1, \ldots, k-1
$$


onde $\theta_{i+}=\sum_{j=1}^{k} \theta_{i j}$ e $\theta_{+i}=\sum_{j=1}^{k} \theta_{j i}$.

Para a construção do FBST, atribuímos uma medida de probabilidade a priori sobre $\Theta$, um conjunto simplex $k^{2}-1$ dimensional. A distribuição Dirichlet é utilizada usualmente como priori para $\boldsymbol{\theta}$, pois seu suporte coincide com a região definida pelo espaço paramétrico além de ser conjugada da distribuição Multinomial (Pereira e Viana, 1982). Supondo que $\boldsymbol{\theta} \sim \operatorname{Dirichlet}(\boldsymbol{\alpha})$, temos que $\boldsymbol{\theta} \mid \boldsymbol{x} \sim \operatorname{Dirichlet}(\boldsymbol{\alpha}+\boldsymbol{x})$. Mais adiante, a fim de obter comparações com resultados clássicos, definimos $\alpha_{i j}=1, \forall i, j \in\{1, \ldots, k\}$, já que, sob essas condições, a moda da posteriori de $\boldsymbol{\theta}$ coincide com seu estimador de máxima verossimilhança.

\subsection{Formulação do FBST}

Nesta seção, descrevemos as técnicas utilizadas para calcular o e-valor em favor da hipótese de homogeneidade marginal para um tabela de contingência bidimensional $k \times k, k \geq 2$. Denotamos por $\Theta_{S}$ e $\Theta_{M}$ os subconjuntos do espaço paramétrico que caracterizam as hipóteses de simetria e homogeneidade marginal, respectivamente, isto é, $\Theta_{S}=\left\{\boldsymbol{\theta} \in \Theta: \theta_{i j}=\theta_{j i}, \forall i, j=1, \ldots, k\right\}$ e $\Theta_{M}=\left\{\boldsymbol{\theta} \in \Theta: \theta_{i+}=\theta_{+i}, \forall i=1, \ldots, k-1\right\}$. Fixado $x \in \mathcal{X}$, a primeira etapa do FBST consiste em obter o máximo da função densidade a posteriori $f(\boldsymbol{\theta} \mid \boldsymbol{x})$, onde

$$
f(\boldsymbol{\theta} \mid \boldsymbol{x}) \propto \prod_{i=1}^{k} \prod_{j=1}^{k} \theta_{i j}^{n_{i j}+\alpha_{i j}-1}
$$

Vale notar que apesar de considerarmos fixado de antemão o tamanho amostral e adotarmos o modelo Multinomial como distribuição amostral para desenvolver a densidade a posteriori (Equação 3.2), a condução de experimentos com outras regras de parada não-informativas em processos de Bernoulli produziriam a mesma inferência sobre $\boldsymbol{\theta}$ devido à proporcionalidade de tais funções de verossimilhança.

Além disso, vale ressaltar que maximizar a Equação 3.2 equivale a maximizar o logaritmo da densidade a posteriori, isto é,

$$
\operatorname{Maximizar} \sum_{i=1}^{k} \sum_{j=1}^{k}\left(n_{i j}+\alpha_{i j}-1\right) \log \theta_{i j}
$$

sujeito às $(k-1)$ restrições

$$
\theta_{i+}=\theta_{+i}, \quad i=1, \ldots, k-1,
$$

além da restrição $\sum_{i, j=1}^{k} \theta_{i j}=1$.

Para tanto, faremos uso do método dos multiplicadores de Lagrange (Bertsekas, 1996). Denotando o vetor de multiplicadores de Lagrange por $\boldsymbol{\lambda}=\left(\lambda_{0}, \lambda_{1}, \ldots, \lambda_{k-1}\right)$, maximizamos a função $\mathcal{L}$ dada por 


$$
\mathcal{L}(\boldsymbol{\theta}, \boldsymbol{\lambda})=\sum_{i=1}^{k} \sum_{j=1}^{k}\left(n_{i j}+\alpha_{i j}-1\right) \log \theta_{i j}-\lambda_{0}\left(\sum_{i=1}^{k} \sum_{j=1}^{k} \theta_{i j}-1\right)-\sum_{l=1}^{k-1} \lambda_{l}\left(\theta_{l .}-\theta_{. l}\right) .
$$

Com isso, as derivadas parciais são:

$$
\begin{aligned}
& \frac{\partial \mathcal{L}(\boldsymbol{\theta}, \boldsymbol{\lambda})}{\theta_{i j}}=\frac{\left(n_{i j}+\alpha_{i j}-1\right)}{\theta_{i j}}-\lambda_{0}-\left(\lambda_{i}-\lambda_{j}\right), \quad \forall i, j=1, \ldots, k, \\
& \frac{\partial \mathcal{L}(\boldsymbol{\theta}, \boldsymbol{\lambda})}{\lambda_{l}}=-\left(\theta_{l .}-\theta_{. l}\right), \quad \forall l=1, \ldots, k-1, \mathrm{e} \\
& \frac{\partial \mathcal{L}(\boldsymbol{\theta}, \boldsymbol{\lambda})}{\lambda_{0}}=-\left(\sum_{i=1}^{k} \sum_{j=1}^{k} \theta_{i j}-1\right) .
\end{aligned}
$$

Igualando-as a zero, obtemos o seguinte sistema de equações

$$
\begin{aligned}
\left(n_{i j}+\alpha_{i j}-1\right) & =\theta_{i j}\left(\lambda_{0}+\lambda_{i}-\lambda_{j}\right), \quad \forall i, j=1, \ldots, k, \\
\theta_{i .} & =\theta_{. i} \quad, \forall i=1, \ldots, k-1, \mathrm{e} \\
\sum_{i=1}^{k} \sum_{j=1}^{k} \theta_{i j} & =1 .
\end{aligned}
$$

Manipulando essas expressões, obtemos $\tilde{\lambda}_{0}=n, \tilde{\theta}_{i i}=\frac{n_{i i}+\alpha_{i i}-1}{n}, i=1, \ldots, k$, e

$$
\tilde{\theta}_{i j}=\frac{n_{i j}+\alpha_{i j}-1}{n+\lambda_{i}-\lambda_{j}}
$$

sujeito a

$$
\sum_{\substack{j=1 \\ j \neq i}}^{k} \frac{n_{i j}+\alpha_{i j}-1}{n+\lambda_{i}-\lambda_{j}}=\sum_{\substack{j=1 \\ j \neq i}}^{k} \frac{n_{j i}+\alpha_{j i}-1}{n+\lambda_{j}-\lambda_{i}}
$$

$i, j=1, \ldots, k$. A expressão 3.4 permite obter os multiplicadores de Lagrange independentemente de $\boldsymbol{\theta}$ e, pela Equação 3.3, determinar a moda da posteriori de $\boldsymbol{\theta} \mid \boldsymbol{x}$ sob $H^{M}$. A unicidade do máximo sob $H^{M}$ segue do fato da log-posteriori ser côncava (assumindo que $n_{i j}+\alpha_{i j}-1>0, \forall i, j=1, \ldots k$ ) e da restrição da hipótese formar um conjunto convexo (Bertsekas, 1996).

Após obter a máxima densidade a posteriori sob $H^{M}$, devemos determinar o conjunto $T_{\boldsymbol{x}}$ de pontos do espaço paramétrico cujas densidades superam esse valor, denominado região tangente à hipótese nula, além de calcular sua probabilidade a posteriori $P\left(\boldsymbol{\theta} \in T_{\boldsymbol{x}} \mid \boldsymbol{x}\right)$. Para avaliar essa probabilidade, geramos $M$ valores da distribuição a posteriori, $\boldsymbol{\theta}_{\mathbf{1}}, \boldsymbol{\theta}_{\mathbf{2}}, \ldots, \boldsymbol{\theta}_{\boldsymbol{M}}$, e comparamos suas funções densidades com o valor máximo avaliado sob $H^{M}$. Assim, sejam $\boldsymbol{\theta}^{*}=\arg \max \{f(\boldsymbol{\theta} \mid \boldsymbol{x})$ : $\left.\boldsymbol{\theta} \in \Theta_{M}\right\}$ e 


$$
\mathbb{I}_{i}= \begin{cases}1, & \text { se } \\ 0, & f\left(\boldsymbol{\theta}_{\boldsymbol{i}} \mid \boldsymbol{x}\right) \geq f\left(\boldsymbol{\theta}^{*} \mid \boldsymbol{x}\right), \\ \text { caso contrário, }\end{cases}
$$

para $i=1, \ldots, M$. Admitindo independência dos pontos simulados $\boldsymbol{\theta}_{\mathbf{1}}, \ldots, \boldsymbol{\theta}_{\boldsymbol{M}}$, temos, pela lei forte dos grandes números, que

$$
\frac{\sum_{i=1}^{M} \mathbb{I}_{i}}{M} \stackrel{q . c .}{\longrightarrow} P\left(\boldsymbol{\theta} \in T_{\boldsymbol{x}} \mid \boldsymbol{x}\right)
$$

Deste modo, aproximamos a evidência de Pereira-Stern a favor da hipótese de homogeneidade marginal através do método de Monte Carlo, ou seja,

$$
\tilde{e v}\left(\Theta_{M} ; \boldsymbol{x}\right)=1-\frac{\sum_{i=1}^{M} \mathbb{I}_{i}}{M} .
$$

No Exemplo 3.1, a evidência a favor da homogeneidade marginal é, aproximadamente, $e v\left(\Theta_{M} ; \boldsymbol{x}\right)=$ 0.68 , enquanto que para simetria é $e v\left(\Theta_{S} ; \boldsymbol{x}\right)=0.20$. Assim, concluímos que os dados trazem consideravelmente mais evidência para suportar homogeneidade marginal do que simetria.

\subsection{Relações com a hipótese de simetria}

É fácil ver que as hipóteses de homogeneidade marginal e simetria são equivalentes para $k=2$. Contudo, essa afirmação não é válida para casos em que $k>2$, pois $\Theta^{S} \subset \Theta^{M}$ (Agresti, 2013). Quando $k>2$,

$$
\Theta_{S} \subseteq \Theta_{M} \Rightarrow T_{x}^{S} \supseteq T_{x}^{M} \Rightarrow e v\left(\Theta_{S} ; x\right) \leq e v\left(\Theta_{M} ; x\right)
$$

Essa relação entre as medidas de evidência para hipóteses aninhadas denomina-se coerência ou monotonicidade (Gabriel, 1969; Izbicki, 2010). Para ilustrar que a hipótese de simetria é mais restritiva do que a hipótese de homogeneidade marginal, apresentamos o Exemplo 3.2.

Exemplo 3.2. Considere a tabela de contingência $3 \times 3$ dada em 3.2 e que desejamos testar as hipóteses de simetria e homogeneidade marginal. Considere que $\boldsymbol{X} \mid \boldsymbol{\theta} \sim \operatorname{Multinomial}(n ; \boldsymbol{\theta})$ e $\boldsymbol{\theta} \sim \operatorname{Dirichlet}(1,1, \ldots, 1)$.

Tabela 3.2: Tabela $3 \times 3$ sob $H M$

\begin{tabular}{ccccc}
\hline & \multicolumn{3}{c}{$\mathrm{Y}$} & \\
$\mathrm{X}$ & $\mathrm{A}$ & $\mathrm{B}$ & $\mathrm{C}$ & Total \\
\cline { 2 - 4 } $\mathrm{A}$ & 4 & 6 & 2 & 12 \\
$\mathrm{~B}$ & 1 & 5 & 8 & 14 \\
$\mathrm{C}$ & 7 & 3 & 4 & 14 \\
Total & 12 & 14 & 14 & 40 \\
\hline
\end{tabular}

Denotando por $\hat{\boldsymbol{\theta}}_{S}$ e $\hat{\boldsymbol{\theta}}_{M}$ as modas da posteriori sob $\Theta_{S}$ e $\Theta_{M}$, respectivamente, e por $\hat{\boldsymbol{\theta}}$ a moda da posteriori em $\Theta$. Assim, 


$$
\hat{\boldsymbol{\theta}}=\frac{1}{40}(4,6,2,1,5,8,7,3,4) .
$$

É possível mostrar que a moda da posteriori em $\Theta_{S}$ é

$$
\hat{\boldsymbol{\theta}}_{S}=\frac{1}{80}(8,7,9,7,10,11,9,11,8)
$$

Observe que a moda da posteriori $\hat{\boldsymbol{\theta}}$ satisfaz a condição de homogeneidade marginal, apesar de não satisfazer a condição de simetria. Assim,

$$
f\left(\hat{\boldsymbol{\theta}}_{S}\right)<f(\hat{\boldsymbol{\theta}})=f\left(\hat{\boldsymbol{\theta}}_{M}\right) .
$$

Logo, o valor da densidade a posteriori em $\hat{\boldsymbol{\theta}}_{\boldsymbol{M}}$ é maior que em $\hat{\boldsymbol{\theta}}_{\boldsymbol{S}}$. Além disso,

$$
T_{x}^{M}=\left\{\boldsymbol{\theta} \in \Theta: f(\boldsymbol{\theta} \mid \boldsymbol{x})>\sup _{\boldsymbol{\theta} \in \Theta_{M}} f(\boldsymbol{\theta} \mid \boldsymbol{x})\right\}=\emptyset .
$$

Consequentemente,

$$
e v\left(\Theta_{M} ; \boldsymbol{x}\right)=1-P\left(\theta \in T_{\boldsymbol{x}} \mid \boldsymbol{x}\right)=1 .
$$

Já, para a hipótese de simetria,

$$
T_{x}^{S}=\left\{\boldsymbol{\theta} \in \Theta: f(\boldsymbol{\theta} \mid \boldsymbol{x})>\sup _{\boldsymbol{\theta} \in \Theta_{S}} f(\boldsymbol{\theta} \mid \boldsymbol{x})\right\} \neq \emptyset .
$$

Além disso,

$$
e v\left(\Theta_{S} ; \boldsymbol{x}\right)=1-P\left(\boldsymbol{\theta} \in T_{\boldsymbol{x}} \mid \boldsymbol{x}\right) \Rightarrow e v\left(\Theta_{S} ; \boldsymbol{x}\right)=0.22<1 .
$$

A evidência 3.8 foi obtida computacionalmente por meio da rotina do Apêndice B.

\subsection{Abordagem clássica ao teste de homogeneidade marginal}

Sob a perspectiva clássica, Lehmann e Romano (2005) mostram que, sob certas restrições, o teste exato de McNemar (Seção 2.3), para hipóteses bilaterais, é não-viesado uniformemente mais poderoso (UMPU) de nível $\alpha, 0<\alpha<1$. Contudo, não existe teste UMPU para verificar a homogeneidade marginal quando $k>2$. Nessa situação, uma abordagem consiste em considerar 
outras classes de testes que podem não possuir resultados de otimalidade desejáveis. Vale notar que não há propostas na literatura em que a estatística de teste possui distribuição exata conhecida para investigar a homogeneidade marginal quando $k>2$. Consideramos, ao longo desta seção, a proposta de Stuart (1953), embora outras alternativas possam ser encontradas na literatura, como, por exemplo, em Madansky (1963) e Bhapkar (1966).

Uma estatística de teste bastante natural para avaliar homogeneidade marginal consiste em tomar o vetor de diferenças das correspondentes contagens marginais $\boldsymbol{d}=\left(d_{1}, d_{2}, \ldots, d_{k-1}\right)$, em que $d_{i}=n_{i+}-n_{+i}, i=1, \ldots, k-1$. A estatística de Stuart-Maxwell é definida, para o ponto amostral $\boldsymbol{x} \in \mathcal{X}$, por

$$
Q(\boldsymbol{x})=\sum_{i, j=1}^{k-1} a_{i j} d_{i} d_{j}
$$

onde $a_{i j}$ é o elemento que ocupa a posição $(i, j)$ da inversa da matriz de covariâncias $V=\left(a_{i j}\right)_{i, j=1}^{k-1}$, tal que

$$
v_{i j}=\left\{\begin{array}{l}
n_{i+}+n_{+i}-2 n_{i i} \quad \text { se } i=j=1, \ldots, k-1, \\
-\left(n_{i j}+n_{j i}\right), \quad \text { se } i \neq j \in\{1, \ldots, k-1\} .
\end{array}\right.
$$

Quando $k=2, Q(\boldsymbol{x})$ se reduz à estatística qui-quadrado de McNemar. Ademais, é possível mostrar que, sob a hipótese $H^{M}, Q(\boldsymbol{X})$ possui, aproximadamente, distribuição qui-quadrado com $k-1$ graus de liberdade (Stuart, 1953). Contudo, quando a tabela contiver diversas contagens baixas, os métodos assintóticos podem ser imprecisos (Basso et al., 2009). Nesse caso, podemos recorrer a métodos de Monte Carlo para obter uma aproximação da função de distribuição empírica de $Q(\boldsymbol{x})$ sob $H^{M}$ e, posteriormente, obter um p-valor empírico (ver detalhes em Davison e Hinkley (1997)). A obtenção de um p-valor exato requer o cálculo de $Q(\boldsymbol{x})$ para todas as possíveis configurações de tabelas de contingência que produzem o tamanho amostral fixado $n$, o que totaliza $|\mathcal{X}|=\left(\begin{array}{c}n+k^{2}-1 \\ k^{2}-1\end{array}\right)$ operações desta natureza. Obviamente, quando $n$ aumenta, o número de configurações torna-se consideravelmente alto, como ilustrado na Tabela 3.3. Deste modo, a obtenção de um p-valor exato se torna computacionalmente infactível e as propostas usuais residem em métodos empíricos baseados em reamostragem (Good, 2005).

Tabela 3.3: Número total de tabelas $k \times k$ para um tamanho amostral $n$

\begin{tabular}{c|c|c|c}
\hline$n$ & $k=3$ & $k=4$ & $k=5$ \\
\hline 20 & $3,11 \times 10^{6}$ & $3,247 \times 10^{9}$ & $1,76 \times 10^{12}$ \\
30 & $4,80 \times 10^{7}$ & $3,45 \times 10^{11}$ & $1,40 \times 10^{15}$ \\
50 & $1,93 \times 10^{9}$ & $2,07 \times 10^{14}$ & $1,75 \times 10^{19}$ \\
100 & $3,52 \times 10^{11}$ & $2,39 \times 10^{18}$ & $2,60 \times 10^{25}$ \\
1000 & $2,57 \times 10^{19}$ & $8,62 \times 10^{32}$ & $2,17 \times 10^{48}$ \\
5000 & $9,76 \times 10^{24}$ & $2,39 \times 10^{43}$ & $1,02 \times 10^{65}$ \\
\hline
\end{tabular}




\subsection{O Modelo Logístico-Normal}

Nesta seção, exploramos uma aproximação do modelo Dirichlet pelo modelo Logístico-Normal, assim como mencionado em Lindley (1964) e Aitchison e Shen (1980). Mais precisamente, desenvolvemos o e-valor para testar homogeneidade marginal considerando o modelo Logístico-Normal, além de compará-lo com os resultados obtidos segundo o modelo Dirichlet. A utilização de tal aproximação pode contribuir substancialmente para a redução do custo computacional no cálculo do e-valor (Rodrigues, 2006).

O modelo logístico-normal pode ser obtido através da transformação logística (ou logito) das componentes de um vetor aleatório com distribuição Normal multivariada. Assim, seja $\boldsymbol{X}$ um vetor aleatório em $\mathbb{R}^{v}$ distribuído, condicionalmente a $\boldsymbol{\mu}$ e $\Sigma, N_{v}(\boldsymbol{\mu}, \Sigma)$, onde $\boldsymbol{\mu}$ é um vetor de médias v-dimensional e $\Sigma$ a matriz $v \times v$ de covariâncias. Considere a transformação

$$
\boldsymbol{\theta}(\boldsymbol{X})=\left(\left(\frac{\exp \left(X_{1}\right)}{1+\sum_{i=1}^{v} \exp \left(X_{i}\right)}\right), \ldots,\left(\frac{\exp \left(X_{v}\right)}{1+\sum_{i=1}^{v} \exp \left(X_{i}\right)}\right)\right)
$$

de $\mathbb{R}^{v}$ em $\mathbb{S}^{v}$, onde $\mathbb{S}^{v}$ representa o conjunto simplex $v$-dimensional $\left\{\left(\mu_{1}, \ldots, \mu_{v}\right) \in \mathbb{R}_{+}^{v}: \mu_{1}+\right.$ $\left.\ldots+\mu_{v} \leq 1\right\}$. É possível mostrar que $\boldsymbol{\theta}(\boldsymbol{X})$ possui distribuição logística-normal (Aitchison e Shen, 1980), cuja densidade, $q(\cdot \mid \boldsymbol{\mu}, \Sigma)$, é dada por

$$
q(\boldsymbol{\theta} \mid \boldsymbol{\mu}, \Sigma)=\frac{1}{(2 \pi)^{v / 2}|\Sigma|^{1 / 2}} \exp \left(-\frac{1}{2}(\operatorname{logit}(\boldsymbol{\theta})-\boldsymbol{\mu})^{T} \Sigma^{-1}(\operatorname{logit}(\boldsymbol{\theta})-\boldsymbol{\mu})\right) \mathbb{I}_{\mathcal{S}^{v}}(\boldsymbol{\theta}),
$$

em que $\operatorname{logit}(\boldsymbol{\theta})=\left(\log \left(\frac{\theta_{1}}{\theta_{v+1}}\right), \ldots, \log \left(\frac{\theta_{v}}{\theta_{v+1}}\right)\right)$ e $\theta_{v+1}=1-\sum_{j=1}^{v} \theta_{j}$.

Lindley (1964) e Aitchison e Shen (1980) propõem uma aproximação da distribuição Dirichlet por uma logística-normal. A similaridade dessas distribuições pode ser investigada por meio de medidas de divergência entre medidas de probabilidade, dentre as quais destacamos a medida de divergência de Kullback-Leibler (Kullback, 1959). Denotando por $p(\cdot \mid \boldsymbol{\alpha})$ a densidade Dirichlet $(\boldsymbol{\alpha})$, a medida de divergência de Kullback-Leibler entre $p(\cdot \mid \boldsymbol{\alpha})$ e $q(\cdot \mid \boldsymbol{\mu}, \Sigma)$ com relação a $p(\cdot \mid \boldsymbol{\alpha}), K(p, q)$, fica dada por

$$
K(p, q)=\int_{\mathcal{S}^{v}} p(\boldsymbol{\theta} \mid \boldsymbol{\alpha}) \log \left(\frac{p(\boldsymbol{\theta} \mid \boldsymbol{\alpha})}{q(\boldsymbol{\theta} \mid \boldsymbol{\mu}, \Sigma)}\right) d \boldsymbol{\theta}
$$

Deste modo, devemos obter o modelo logístico-normal que minimiza $K(p, q)$ em relação a $\boldsymbol{\mu}$ e $\Sigma$. Pode-se mostrar que a Equação 3.11 é proporcional a

$$
\log |\Sigma|+\int_{\mathcal{S}^{v}}(\operatorname{logit}(\boldsymbol{\theta})-\boldsymbol{\mu})^{T} \Sigma^{-1}(\operatorname{logit}(\boldsymbol{\theta})-\boldsymbol{\mu}) p(\boldsymbol{\theta} \mid \boldsymbol{\alpha}) d \boldsymbol{\theta}
$$

que é minimizada quando 


$$
\boldsymbol{\mu}=E(\operatorname{logit}(\boldsymbol{\theta})) \text { e } \Sigma=\operatorname{Var}(\operatorname{logit}(\boldsymbol{\theta}))
$$

ambas calculadas em relação a $p(\cdot \mid \boldsymbol{\alpha})$. Ademais, pela relação entre as distribuições Gama e Dirichlet, segue que

$$
\begin{gathered}
E\left(\operatorname{logit}\left(\theta_{i}\right)\right)=\psi\left(\alpha_{i}\right)-\psi\left(\alpha_{v+1}\right), \\
\operatorname{Var}\left(\operatorname{logit}\left(\theta_{i}\right)\right)=\psi^{\prime}\left(\alpha_{i}\right)+\psi^{\prime}\left(\alpha_{v+1}\right) \text { e } \\
\operatorname{Cov}\left(\operatorname{logit}\left(\theta_{i}\right), \operatorname{logit}\left(\theta_{j}\right)\right)=\psi^{\prime}\left(\alpha_{v+1}\right),
\end{gathered}
$$

onde $\psi(\cdot)=\frac{\Gamma^{\prime}(\cdot)}{\Gamma(\cdot)}$. Considere agora $\boldsymbol{X} \mid \boldsymbol{\theta} \sim \operatorname{Multinomial}(n, \boldsymbol{\theta})$ e $\boldsymbol{\theta} \sim \operatorname{Dirichlet}(\boldsymbol{\alpha})$. Logo, $\boldsymbol{\theta} \mid \boldsymbol{x} \sim$ Dirichlet $(\boldsymbol{z})$, tal que $\boldsymbol{z}=\boldsymbol{\alpha}+\boldsymbol{x}$. Utilizando a transformação logística multivariada, sabemos que o vetor

$$
\boldsymbol{\tau}=\left(\tau_{1}, \tau_{2}, \ldots, \tau_{v}\right)=\left(\log \left(\frac{\theta_{1}}{\theta_{v+1}}\right), \log \left(\frac{\theta_{2}}{\theta_{v+1}}\right), \ldots, \log \left(\frac{\theta_{v}}{\theta_{v+1}}\right)\right)
$$

tem distribuição aproximadamente normal $v$-dimensional com vetor de médias

$$
\boldsymbol{\mu}=\left[\begin{array}{c}
\psi\left(z_{1}\right)-\psi\left(z_{v+1}\right) \\
\psi\left(z_{2}\right)-\psi\left(z_{v+1}\right) \\
\vdots \\
\psi\left(z_{v}\right)-\psi\left(z_{v+1}\right)
\end{array}\right]
$$

e matriz de covariâncias

$$
\boldsymbol{\Sigma}=\left[\begin{array}{cccc}
\psi^{\prime}\left(z_{1}\right)+\psi^{\prime}\left(z_{v+1}\right) & \psi^{\prime}\left(z_{v+1}\right) & \cdots & \psi^{\prime}\left(z_{v+1}\right) \\
\psi^{\prime}\left(z_{v}\right) & \psi^{\prime}\left(z_{2}\right)+\psi^{\prime}\left(z_{v+1}\right) & \cdots & \psi^{\prime}\left(z_{v+1}\right) \\
& \vdots & \ddots & \\
\psi^{\prime}\left(z_{v+1}\right) & \psi^{\prime}\left(z_{v+1}\right) & \cdots & \psi^{\prime}\left(z_{v}\right)+\psi^{\prime}\left(z_{v+1}\right)
\end{array}\right]
$$

Como estamos aplicando tranformação $\tau$ injetora sobre o espaço paramétrico original, vamos adotar uma densidade de referência para desenvolver a formulação do FBST invariante sob reparametrizações (Madruga et al., 2002). Estabelecemos a distribuição Dirichlet $(1,1, \ldots, 1)$ como a densidade referência, denotada por $r(\boldsymbol{\theta})$. Logo, a densidade referência transformada, $r(\boldsymbol{\tau})$, é aproximadamente normal $v$-dimensional com vetor de médias $\boldsymbol{\mu}=\mathbf{0}$ e matriz de covariâncias:

$$
\boldsymbol{\Sigma}_{r}=\left[\begin{array}{cccc}
2 \psi^{\prime}(1) & \psi^{\prime}(1) & \cdots & \psi^{\prime}(1) \\
\psi^{\prime}(1) & 2 \psi^{\prime}(1) & \cdots & \psi^{\prime}(1) \\
& \vdots & \ddots & \\
\psi^{\prime}(1) & \psi^{\prime}(1) & \cdots & 2 \psi^{\prime}(1)
\end{array}\right]
$$


Consequentemente, a função surpresa relativa é dada por

$$
g(\boldsymbol{\tau})=\frac{f(\boldsymbol{\tau} \mid \boldsymbol{x})}{r(\boldsymbol{\tau})}=|\Sigma|^{-1 / 2}\left|\Sigma_{r}\right|^{1 / 2} \exp \left[-\frac{1}{2}\left((\boldsymbol{\tau}-\boldsymbol{\mu})^{T} \Sigma^{-1}(\boldsymbol{\tau}-\boldsymbol{\mu})-\boldsymbol{\tau}^{T} \Sigma_{r}^{-1} \boldsymbol{\tau}\right)\right] .
$$

Obtemos então o conjunto tangente de forma análoga ao que foi desenvolvido quando adotamos o modelo Dirichlet, embora aqui a geração dos pontos seja feita com base na distribuição normal multivariada.

Nos próximos exemplos, comparamos as evidências obtidas segundo o modelo Dirichlet e o modelo Logístico Normal ao perturbarmos uma das caselas de uma tabela $2 \times 2$ e de uma tabela $3 \times 3$.

Exemplo 3.3. Considere a tabela de contingência $2 \times 2$ :

Tabela 3.4: Tabela de contingência $2 \times 2$

\begin{tabular}{cccc}
\hline & & $\mathrm{Y}$ & \\
$\mathrm{X}$ & 1 & 2 & Total \\
\cline { 2 - 4 } 1 & 20 & $x$ & $20+x$ \\
2 & $20-x$ & 15 & $35-x$ \\
Total & $40-x$ & $15+x$ & 55 \\
\hline
\end{tabular}

$x \in\{0, \ldots, 20\}$. Apresentamos os e-valores para testar homogeneidade marginal. Tais valores foram obtidos tanto pelo modelo Logístico-Normal quanto pelo modelo Dirichlet e são apresentados na Figura 3.1.

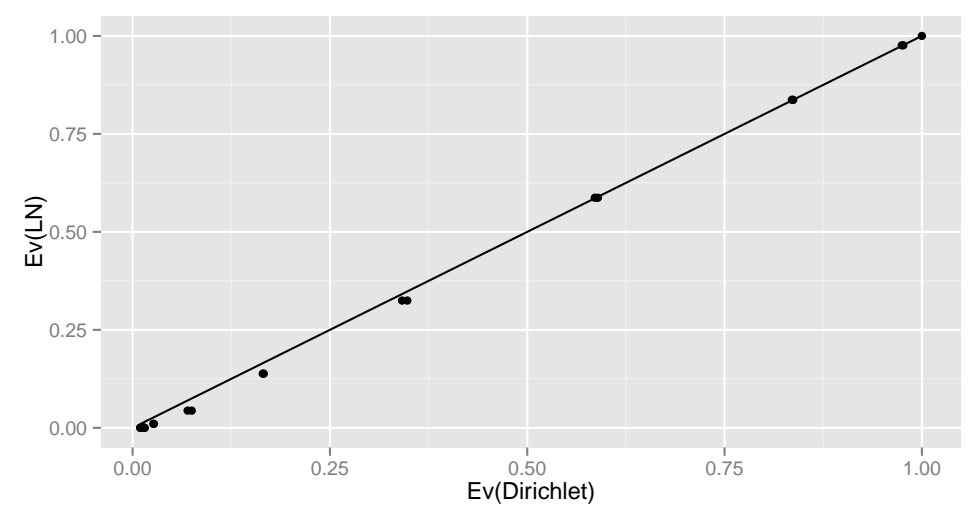

Figura 3.1: Evidências de homogeneidade marginal para os modelos Dirichlet e Logístico-Normal

As evidências obtidas segundo os dois modelos são relativamente similares, além de não afetarem na decisão de rejeitar ou aceitar a hipótese de homogeneidade marginal. A maior diferença absoluta entre as duas evidências é de $3,1 \%$, que ocorre para $x=4$. Para cada $x \in\{0, \ldots, 20\}$, calculamos as medidas de divergência de Kullback-Leibler (Figura 3.2). Note, pela Figura 3.2, que a aproximação é mais precisa em casos que favorecem a hipótese testada.

Exemplo 3.4. Considere a tabela de contingência $3 \times 3$ :

$x \in\{1,2, \ldots, 29\}$. Os resultados dos e-valores para testar homogeneidade marginal segundo os modelos Dirichlet e logístico-normal são apresentados na Figura 3.3. Já as medidas de divergência de Kullback-Leibler de acordo com $x \in\{1, \ldots, 29\}$ são dadas na Figura 3.4.

As evidências obtidas segundo os modelos Dirichlet e Logístico-Normal são relativamente similares, 


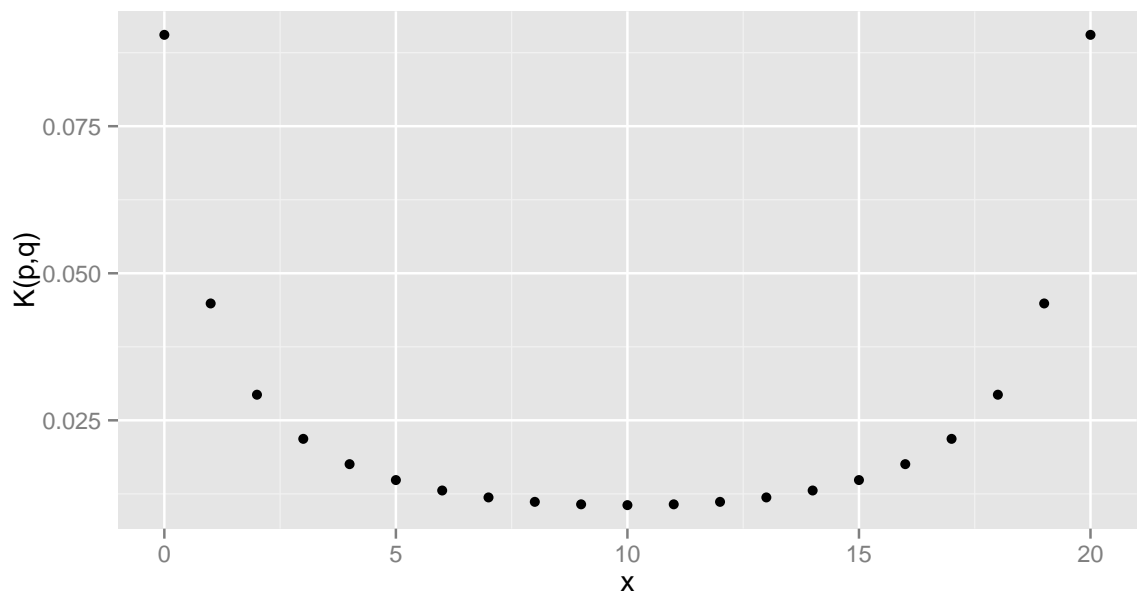

Figura 3.2: Medidas de Kullback-Leibler para as tabelas $2 x 2$ geradas

Tabela 3.5: Tabela de contingência $3 \times 3$

\begin{tabular}{ccccc}
\hline & & $\mathrm{Y}$ & & \\
$\mathrm{X}$ & 1 & 2 & 3 & Total \\
\cline { 2 - 4 } 1 & 15 & 30 & $x$ & $45+x$ \\
2 & 30 & 10 & 15 & 45 \\
3 & $30-x$ & 15 & 10 & $55-x$ \\
Total & $65-x$ & 55 & $25+x$ & 145 \\
\hline
\end{tabular}

além de não acarretarem em mudanças de decisão de rejeitar ou aceitar a hipótese de homogeneidade marginal. A maior diferença absoluta é de $5,1 \%$ e ocorre para $x=1$. Note também, pela Figura 3.4, que a aproximação é mais precisa em casos que favorecem a hipótese de homogeneidade marginal. Apesar das evidências do modelo Dirichlet e Logístico-Normal serem próximas nesses exemplos específicos, existem situações em que tal aproximação pode não ser satisfatória, como quando a posteriori Dirichlet possui parâmetros $\alpha_{i}<5$ (Aitchison e Shen, 1980). 


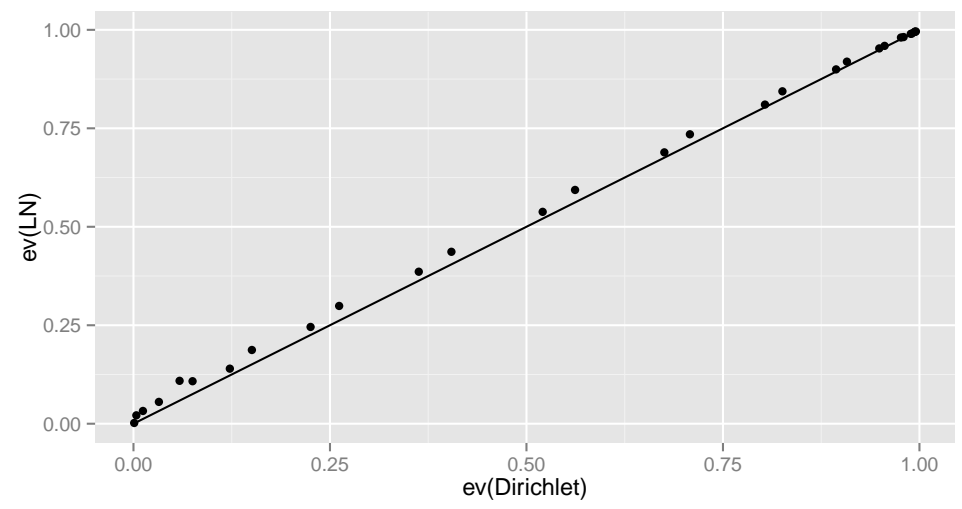

Figura 3.3: Evidências de homogeneidade marginal para os modelos Dirichlet e Logístico-Normal

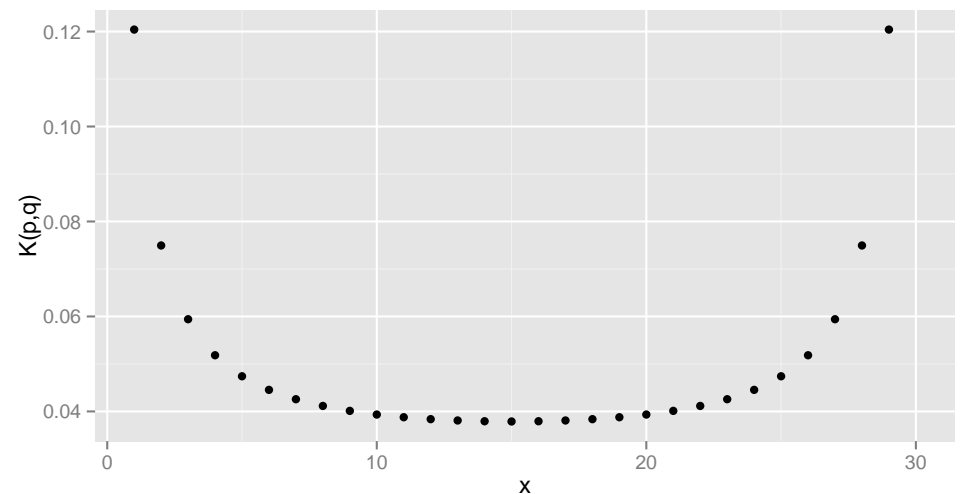

Figura 3.4: Medida de divergência de Kullback-Leibler para as tabelas 3 x 3 geradas

\subsection{Relação entre e-valor e p-valor}

O e-valor (definido na Seção 1.2.3) pode ser visto como uma estatística de teste no FBST, tal como o p-valor para testes de hipóteses sob a abordagem clássica. Assim, nesta seção, vamos avaliar as distribuições amostrais do p-valor e do e-valor e compará-las. Para isto, vamos avaliar a distribuição amostral do e-valor e estabelecer relações com o p-valor. Por exemplo, Diniz et al. (2012) sugerem uma relação funcional entre o p-valor do teste da razão de verossimilhanças com o e-valor do FBST dada por:

$$
e v\left(\Theta_{0} ; \boldsymbol{x}\right) \approx 1-F_{m}\left[F_{m-h}^{-1}(1-p)\right]
$$

onde $F_{m}(\cdot)$ é a função de distribuição acumulada de uma $\chi_{m}^{2}$. Buscando investigar tal relação de uma maneira descritiva, geramos $M=1000$ amostras Multinomiais com parâmetros $n$ ( $n=20$, $50,100,200,500,1000)$ e $\boldsymbol{\theta}_{\boldsymbol{i}}, i=1, \ldots, M$, em que cada ponto $\boldsymbol{\theta}_{\boldsymbol{i}}$ foi gerado de acordo com uma distribuição Dirichlet $(1,1, \ldots, 1)$. Cada quadro da Figura 3.5 representa a relação do e-valor em favor da homogeneidade marginal (3.6) com o p-valor empírico produzido pela estatística de Stuart-Maxwell (3.9), supondo $n$ fixado.

Considerando tamanhos amostrais relativamente pequenos $(n=20,50,100)$, nota-se diferenças consideráveis entre a relação proposta e os pontos gerados $\left(\bar{\Delta}=0,023\right.$ e $\Delta_{\max }=0,38$ para $\left.n=20\right)$. Porém, em tamanhos amostrais moderadamente grandes $(n=200,500,1000)$, os pontos gerados 
se concentram mais próximos da curva proposta $\left(\bar{\Delta}=0,0005\right.$ e $\Delta_{\max }=0,11$ para $\left.n=1000\right)$. Contudo, essas observações são válidas apenas para o contexto $3 \times 3$, pois, em dimensões mais elevadas, a aproximação depende de tamanhos amostrais ainda maiores.
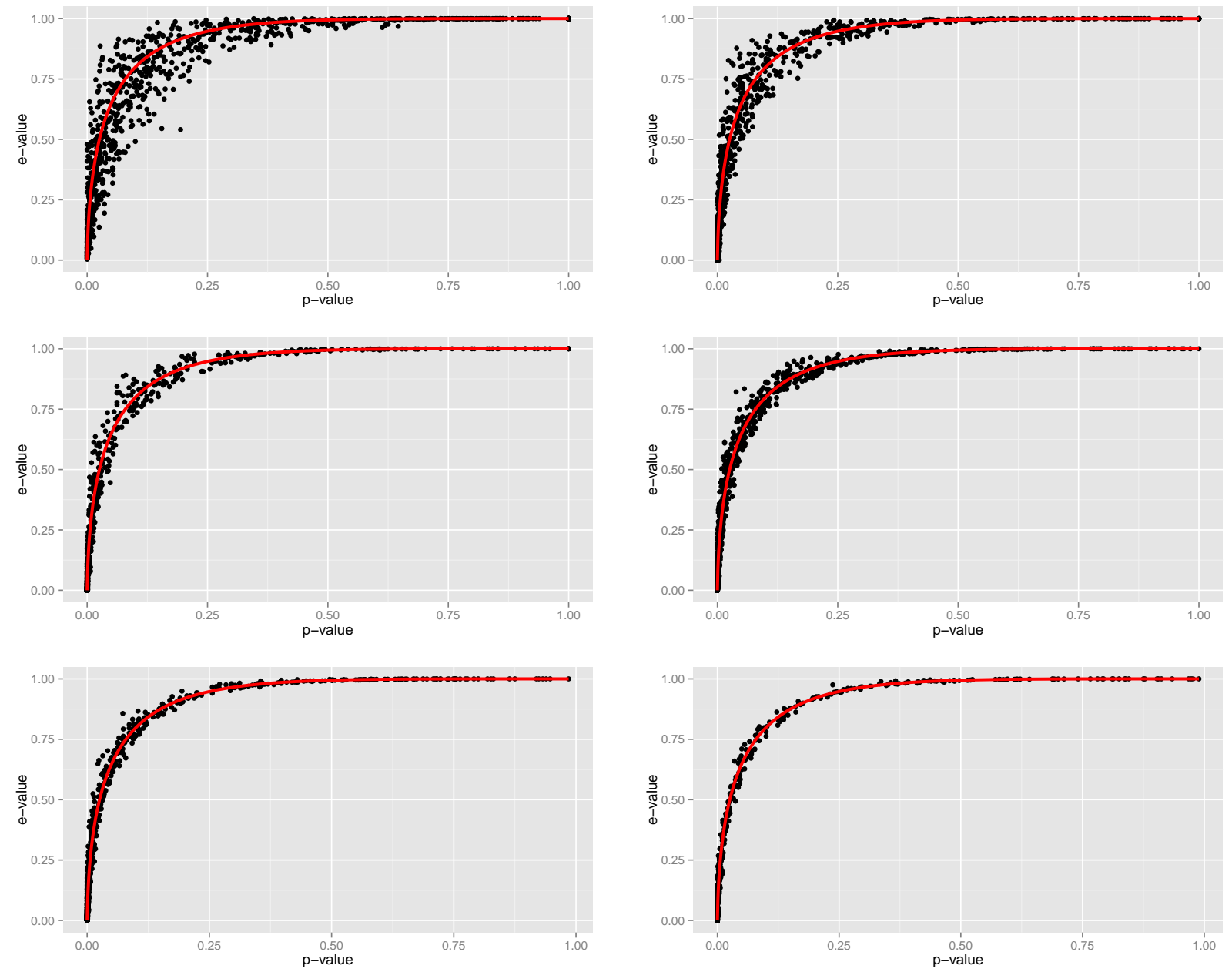

Figura 3.5: Relação entre e-valor e p-valor (teste de homogeneidade marginal em tabelas $3 \times 3$ )

\subsection{Exemplos}

Apresentamos, nesta seção, alguns exemplos de aplicação do FBST em tabelas de contingência para dados reais. Para isto, consideramos que $\boldsymbol{X} \mid \boldsymbol{\theta} \sim \operatorname{Multinomial}(n ; \boldsymbol{\theta})$ e $\boldsymbol{\theta} \sim \operatorname{Dirichlet}(1,1, \ldots, 1)$.

Exemplo 3.5. (Breen e Hayes, 1997) A Tabela 3.6 mostra a religião de uma amostra de indivíduos durante duas sondagens. O conjunto de possíveis religiões é $\mathbb{C}=\{$ Católica (1), Anglicana (2), Protestante Americano (3), Protestante Fundamentalista (4), Outro Protestante (5) ou Nenhuma (6)\}. Um objetivo do estudo consiste em verificar se as distribuições das religiões em ambos os instantes coincidem, o que pode ser descrito pela hipótese de homogeneidade marginal, isto é, $H^{M}: \theta_{i+}=\theta_{+i}, i=1, \ldots, 5$.

O e-valor para a hipótese de homogeneidade marginal, calculado pelos métodos descritos em 3.3, é $e v\left(\Theta_{M} ; \boldsymbol{x}\right)<5 \times 10^{-4}$. Logo, há evidências para concluir que a distribuição das religiões se alterou no segundo momento. Esses resultados concordam com a abordagem clássica, já que o p-valor empírico do teste de Stuart-Maxwell é $p(\boldsymbol{x})<2 \times 10^{-6}$. 
Tabela 3.6: Dados de mobilidade religiosa (Breen e Hayes, 1997)

\begin{tabular}{cccccccc}
\hline & \multicolumn{7}{c}{ Religião atual } \\
\cline { 2 - 6 } Religião inicial & 1 & 2 & 3 & 4 & 5 & 6 & Total (\%) \\
\hline 1 & 123 & 2 & 0 & 0 & 1 & 48 & $174(0,153)$ \\
2 & 10 & 420 & 9 & 1 & 4 & 217 & $661(0,582)$ \\
3 & 2 & 21 & 102 & 1 & 5 & 54 & $185(0,163)$ \\
4 & 0 & 8 & 2 & 15 & 0 & 6 & $31(0,027)$ \\
5 & 0 & 4 & 0 & 0 & 7 & 5 & $16(0,014)$ \\
6 & 1 & 3 & 0 & 1 & 1 & 62 & $68(0,060)$ \\
Total & 136 & 458 & 113 & 18 & 18 & 392 & 1135 \\
$(\%)$ & $(0,120)$ & $(0,403)$ & $(0,099)$ & $(0,016)$ & $(0,016)$ & $(0,345)$ & \\
\hline
\end{tabular}

Exemplo 3.6. (Agresti, 2007) Em uma pesquisa de mercado, registrou-se a marca de café instantâneo adquirida por cada um de 541 clientes em dois períodos. Seja $\mathbb{C}=\{$ Brim, High Point, Nescafe, Sanka, Taster's\} o conjunto das marcas analisadas. Um dos objetivos do estudo consistia em verificar eventuais alterações nos padrões de consumo dos indivíduos durante essas duas ocasiões.

Tabela 3.7: Padrões de consumo de café em dois períodos de compra (Agresti, 2007)

\begin{tabular}{ccccccc}
\hline & \multicolumn{6}{c}{ Segunda compra } \\
\cline { 2 - 6 } Primeira compra & High Point & Taster's & Sanka & Nescafe & Brim & Total (\%) \\
High Point & 93 & 17 & 44 & 7 & 10 & $171(0,316)$ \\
Taster's & 9 & 46 & 11 & 0 & 9 & $75(0,139)$ \\
Sanka & 17 & 11 & 155 & 9 & 12 & $204(0,377)$ \\
Nescafe & 6 & 4 & 9 & 15 & 2 & $36(0,066)$ \\
Brim & 10 & 4 & 12 & 2 & 27 & $55(0,102)$ \\
Total & 135 & 82 & 231 & 33 & 60 & 541 \\
$(\%)$ & $(0,249)$ & $(0,152)$ & $(0,427)$ & $(0,061)$ & $(0,111)$ & \\
\hline
\end{tabular}

Para testar se a opinião dos consumidores permaneceu a mesma na segunda compra, empregamos o teste de homogeneidade marginal. Neste caso, estamos investigando a hipótese $H_{M}: \theta_{i+}=\theta_{+i}, i=$ $1, \ldots, 4$. Calculando o e-valor pelos métodos descritos em 3.3 , obtemos que $e v\left(\Theta_{M} ; \boldsymbol{x}\right)=0.974$, enquanto que o p-valor do teste de Stuart-Maxwell é $p(\boldsymbol{x})=0.013$. Note também que se utilizarmos a relação do e-valor com o p-valor (3.14), obtemos que

$$
e v\left(\Theta_{M} ; \boldsymbol{x}\right) \approx 1-F_{24}\left[F_{4}^{-1}(1-0.013)\right]=0.971 .
$$

Assim, o e-valor aponta que não há evidências de que a opinião dos consumidores se alterou na segunda compra, diferentemente do p-valor que sugere uma conclusão oposta. 


\section{Capítulo 4}

\section{Tabelas multidimensionais}

\subsection{Introdução}

Neste capítulo, apresentamos testes de homogeneidade marginal para tabelas multidimensionais, com a presença de $d$ variáveis observadas, $d>2$. Em tabelas multidimensionais, várias hipóteses de homogeneidade marginal podem ser formuladas, por exemplo, via condicionamento em uma terceira variável. Contudo, como o interesse reside, geralmente, na comparação das marginais univariadas, desenvolvemos apenas esse teste. Exibimos exemplos com tabelas tridimensionais, embora a generalização do teste para tabelas de dimensão maior possa ser feita de maneira análoga.

\subsection{Motivação}

Exemplo 4.1. (Anderson, 1951) Em uma eleição majoritária, realizou-se um estudo para verificar a alteração nos padrões de intenção de votos dos eleitores ao longo de três meses: Junho, Julho e Agosto. Seja $\mathbb{C}=\{$ Republicano (1), Democrata (2), Incerto (3) $\}$ o conjunto de possíveis categorias de resposta dos indivíduos. Os dados estão dispostos na Tabela 4.1 e fazem parte de um estudo da campanha eleitoral de 1940 em Ohio, nos Estados Unidos. Neste caso, o espaço paramétrico e o espaço amostral podem ser representados de forma similar ao do Exemplo 3.2.

Considere que $\boldsymbol{X} \mid \boldsymbol{\theta} \sim \operatorname{Multinomial}(n ; \boldsymbol{\theta})$. Assim, um interesse consiste em investigar a homogeneidade dos padrões de intenção de voto nos três meses, isto é:

$$
H^{M}: \theta_{i++}=\theta_{+i+}=\theta_{++i}, i=1,2,
$$

onde $\theta_{i++}=\sum_{j=1}^{k} \sum_{l=1}^{k} \theta_{i j l}, \theta_{+i+}=\sum_{j=1}^{k} \sum_{l=1}^{k} \theta_{j i l}$ e $\theta_{++i}=\sum_{j=1}^{k} \sum_{l=1}^{k} \theta_{j l i}$.

$\mathrm{Na}$ próxima seção, desenvolvemos o FBST para testar homogeneidade marginal em tabelas tridimensionais.

\subsection{Homogeneidade Marginal em Tabelas Tridimensionais}

De maneira análoga ao Capítulo 3, assumimos que $\boldsymbol{\theta} \sim \operatorname{Dirichlet}(\boldsymbol{\alpha})$. A primeira etapa do FBST consiste em obter a máxima densidade a posteriori sob $H_{M}$. Isto pode ser obtido, equivalentemente, através do logaritmo da densidade a posteriori. Assim, devemos 
Tabela 4.1: Dados de intenção de voto ao longo de Junho, Julho e Agosto (Anderson, 1951)

\begin{tabular}{|c|c|c|c|c|c|}
\hline \multirow[b]{2}{*}{ Junho } & \multicolumn{4}{|c|}{ Agosto } & \multirow[b]{2}{*}{ Total } \\
\hline & Julho & 1 & 2 & 3 & \\
\hline \multirow{5}{*}{ Republicano (1) } & 1 & 120 & 6 & 2 & 128 \\
\hline & 2 & 1 & 2 & 1 & 4 \\
\hline & 3 & 8 & 1 & 7 & 16 \\
\hline & Total & 129 & 9 & 10 & 148 \\
\hline & & 1 & 2 & 3 & Total \\
\hline \multirow{5}{*}{ Democrata (2) } & 1 & 6 & 2 & 1 & 9 \\
\hline & 2 & 2 & 103 & 4 & 109 \\
\hline & 3 & 1 & 5 & 8 & 14 \\
\hline & Total & 9 & 109 & 13 & 131 \\
\hline & & 1 & 2 & 3 & Total \\
\hline \multirow{4}{*}{ Incerto $(3)$} & 1 & 20 & 1 & 2 & 23 \\
\hline & 2 & 3 & 6 & 1 & 10 \\
\hline & 3 & 31 & 30 & 81 & 142 \\
\hline & Total & 54 & 37 & 84 & 175 \\
\hline
\end{tabular}

$\operatorname{Maximizar} \sum_{i=1}^{k} \sum_{j=1}^{k} \sum_{l=1}^{k}\left(n_{i j l}+\alpha_{i j l}-1\right) \log \theta_{i j l}$

sujeito às $2(k-1)$ restrições

$$
\theta_{i++}=\theta_{+i+}=\theta_{++i}, \quad i=1, \ldots, k-1,
$$

além da restrição $\sum_{i=1}^{k} \sum_{j=1}^{k} \sum_{l=1}^{k} \theta_{i j l}=1$. Novamente, fazemos uso do método dos multiplicadores de Lagrange.

Seja $\boldsymbol{\lambda}=\left(\mu, \lambda_{1}, \ldots, \lambda_{k-1}, \nu_{1}, \ldots, \nu_{k-1}\right)$ o vetor de multiplicadores de Lagrange. Com isso, a função lagrangiana é dada por

$$
\begin{aligned}
\mathcal{L}(\boldsymbol{\theta}, \boldsymbol{\lambda}) & =\sum_{i=1}^{k} \sum_{j=1}^{k} \sum_{l=1}^{k}\left(n_{i j l}+\alpha_{i j l}-1\right) \log \theta_{i j l}-\mu\left(\sum_{i=1}^{k} \sum_{j=1}^{k} \sum_{l=1}^{k} \theta_{i j l}-1\right)- \\
& -\sum_{r=1}^{k-1} \lambda_{r}\left(\theta_{r++}-\theta_{+r+}\right)-\sum_{u=1}^{k-1} \nu_{u}\left(\theta_{u++}-\theta_{++u}\right) .
\end{aligned}
$$

Obtendo as derivadas parciais e igualando-as a zero, temos o seguinte sistema de equações:

$$
\tilde{\theta}_{i j l}=\frac{n_{i j l}+\alpha_{i j l}-1}{n+\left(\lambda_{i}-\lambda_{j}\right)+\left(\nu_{i}-\nu_{l}\right)}
$$

sujeito a 


$$
\begin{aligned}
\sum_{j=1}^{k} \sum_{l=1}^{k} \frac{n_{i j l}+\alpha_{i j l}-1}{n+\left(\lambda_{i}-\lambda_{j}\right)+\left(\nu_{i}-\nu_{l}\right)} & =\sum_{j=1}^{k} \sum_{l=1}^{k} \frac{n_{j i l}+\alpha_{j i l}-1}{n+\left(\lambda_{j}-\lambda_{i}\right)+\left(\nu_{j}-\nu_{l}\right)} \\
\sum_{j=1}^{k} \sum_{l=1}^{k} \frac{n_{i j l}+\alpha_{i j l}-1}{n+\left(\lambda_{i}-\lambda_{j}\right)+\left(\nu_{i}-\nu_{k}\right)} & =\sum_{j=1}^{k} \sum_{l=1}^{k} \frac{n_{j l i}+\alpha_{j l i}-1}{n+\left(\lambda_{j}-\lambda_{l}\right)+\left(\nu_{j}-\nu_{i}\right)},
\end{aligned}
$$

que pode ser resolvido através de métodos iterativos como o de Newton-Raphson. Posteriormente, devemos gerar $M$ valores da distribuição a posteriori e comparar suas funções densidades com a máxima avaliada sob $H^{M}$. Assim, seguindo os mesmos passos do caso bidimensional, aproximamos a evidência de Pereira-Stern a favor da hipótese de homogeneidade marginal em tabelas tridimensionais através do método de Monte Carlo.

Note que a medida de evidência do FBST leva à decisão de rejeitar ou não essa hipótese. Contudo, em caso de rejeição da homogeneidade marginal tridimensional, podemos estar interessados em investigar quais são as variáveis que apresentam diferenças significativas. Para isso, faz-se necessário comparar as marginais aos pares separadamente. A Tabela 4.2 mostra os resultados para os testes de homogeneidade marginal tridimensional e bidimensionais do Exemplo 4.1.

Tabela 4.2: Evidência de Pereira-Stern para dados do Exemplo 4.1

\begin{tabular}{rr}
\hline Comparação & e-valor \\
\hline Junho-Julho-Agosto & $8,7 \times 10^{-5}$ \\
Junho-Julho & 0.98 \\
Julho-Agosto & 0.009 \\
Junho-Agosto & 0.010 \\
\hline
\end{tabular}

Consequentemente, podemos concluir que não houveram alterações substanciais nas intenções de voto ao longo do mês de Julho, enquanto que há fortes evidências de que as intenções de voto mudaram no mês de Agosto.

\subsection{Hipóteses Lineares}

Os testes desenvolvidos até o momento possuem uma representação matricial que depende do vetor de parâmetros $\boldsymbol{\theta}$ e de uma matriz $C$. Por exemplo, suponha uma tabela de contingência $3 \times 3 \times 3$ em que desejamos testar a hipótese de homogeneidade marginal, dada por:

$$
H^{M}:\left\{\begin{array}{l}
\theta_{1++}=\theta_{+1+}=\theta_{++1} \\
\theta_{2++}=\theta_{+2+}=\theta_{++2}
\end{array} \Leftrightarrow H^{M}:\left\{\begin{array}{l}
\sum_{i, j=1}^{3}\left(\theta_{1 i j}-\theta_{j 1 i}\right)=0 \\
\sum_{i, j=1}^{3}\left(\theta_{1 i j}-\theta_{j i 1}\right)=0 \\
\sum_{i, j=1}^{3}\left(\theta_{2 i j}-\theta_{j 2 i}\right)=0 \\
\sum_{i, j=1}^{3}\left(\theta_{2 i j}-\theta_{j i 2}\right)=0
\end{array} \Leftrightarrow H^{M}: C \boldsymbol{\theta}=\mathbf{0}\right.\right.
$$

O conjunto de vetores formados pelas linhas da matriz $C$ são linearmente independentes e a soma dos elementos de cada linha é igual a zero ( $C$ é denominada matriz de contrastes).

Com essa representação matricial, é possível generalizar diversos testes para tabelas de contingência se a hipótese a ser testada puder ser representada como uma forma linear em $\boldsymbol{\theta}$. De forma análoga aos casos bidimensional e tridimensional, a maximização da posteriori restrita à hipótese 
$H^{M}$ pode ser feita pelo método dos multiplicadores de Lagrange, resultando em um sistema de equações lineares cuja solução é obtida numericamente.

Exemplo 4.2. (Grizzle et al., 1969) Considere um ensaio clínico com 46 pacientes, em que cada um deles foi submetido aos tratamentos $A, B$ e $C$. O conjunto de respostas possíveis de um paciente a cada tratamento é $\mathbb{C}=\{$ Favorável, Desfavorável $\}$. Os dados encontram-se na Tabela 4.3 .

Tabela 4.3: Respostas aos tratamentos A,B e C (Grizzle et al., 1969)

\begin{tabular}{|c|c|c|c|c|c|c|}
\hline \multirow{4}{*}{ Resposta ao trat. C } & \multicolumn{6}{|c|}{ Resposta ao trat. A } \\
\hline & \multicolumn{2}{|c|}{ Favorável } & \multicolumn{4}{|c|}{ Desfavorável } \\
\hline & \multicolumn{2}{|c|}{ Resposta ao trat. B } & \multicolumn{4}{|c|}{ Resposta ao trat. B } \\
\hline & Favorável & Desfavorável & Total & Favorável & Desfavorável & Total \\
\hline Favorável & 6 & 2 & 8 & 2 & 6 & 8 \\
\hline Desfavorável & 16 & 4 & 20 & 4 & 6 & 10 \\
\hline Total & 22 & 6 & 28 & 6 & 12 & 18 \\
\hline
\end{tabular}

Para avaliar se os tratamentos são equivalentes, podemos testar a hipótese de homogeneidade marginal $H^{M}: \theta_{1++}=\theta_{+1+}=\theta_{++1}$, isto é, se as respostas aos três tratamentos são igualmente distribuídas. Pelos métodos descritos na Seção 4.3, obtemos que o e-valor para testar $H^{M}$ é $e v\left(\Theta_{0} ; \boldsymbol{x}\right)=0.46$. Assim, não há evidências de diferenças entre os tratamentos $A, B$ e $C$. 


\section{Capítulo 5}

\section{Conclusões}

\subsection{Considerações Finais}

Neste trabalho, revisamos alguns procedimentos para testar a homogeneidade de proporções marginais para dados categorizados. Vimos que o cálculo do p-valor para a hipótese de homogeneidade marginal em tabelas $2 \times 2$ pode ser abordado através de uma verossimilhança condicional, que elimina parâmetros de perturbação. Além disso, a derivação do p-valor sob o modelo incondicional é analiticamente intratável. As estatísticas de teste geralmente adotadas para o teste de homogeneidade marginal em tabelas bidimensionais sob a abordagem clássica dependem de resultados assintóticos, baseados na distribuição qui-quadrado, ou em métodos computacionais intensivos (testes de permutação), pois o número de configurações de tabelas aumenta consideravelmente à medida que o número de categorias cresce. Já na abordagem bayesiana usual, é necessário atribuir uma probabilidade a priori positiva para a hipótese precisa de homogeneidade marginal, resultando em uma distribuição a posteriori mista, num certo sentido artificial sob a concepção subjetiva de probabilidade.

O FBST contorna essas dificuldades, ou seja, não depende da eliminação de parâmetros de perturbação (sua definição é dada no espaço paramétrico original) e não requer a probabilidade a priori positiva para a hipótese de interesse como no teste de Jeffreys. Ademais, o cálculo do e-valor é simples de ser efetuado computacionalmente mesmo para o caso de tabelas $k \times k$. Mostramos que é possível obter a moda da posteriori sob homogeneidade marginal apenas numericamente através do método dos multiplicadores de Lagrange, o que minimiza o custo computacional. Nos exemplos apresentados, ilustramos também as inconsistências lógicas advindas do p-valor quando hipóteses aninhadas são consideradas. Tais inconsistências não ocorrem no FBST. Avaliamos ainda a utilização de uma aproximação do modelo Dirichlet pelo modelo Logístico-Normal, o que contribuiu consideravelmente para reduzir o tempo computacional de obtenção do e-valor em alguns problemas com contagens muito elevadas. Discutimos brevemente quando tal aproximação parece ser satisfatória.

Finalmente, desenvolvemos o FBST para testar homogeneidade marginal em tabelas tridimensionais. Vimos que o cálculo do e-valor é análogo ao caso bidimensional e que, após testar a igualdade das marginais simultaneamente, é comum investigar a homogeneidade marginal aos pares. Além disso, os testes de hipóteses considerados neste trabalho podem ser representados como uma forma linear nos parâmetros, assim como diversos outros testes comumente empregados em dados categorizados. 


\subsection{Sugestões para Pesquisas Futuras}

- Desenvolver o FBST para outros testes potencialmente de interesse em tabelas de contingência, como, por exemplo, ao considerar variáveis explicativas. No exemplo de intenção de voto, poderíamos estratificar a amostra segundo a variável região ou classe socioeconômica.

- Estudar o FBST para testes de homogeneidade em cadeias de Markov. No contexto clássico, isso já foi discutido por Madansky (1963).

- Utilizar os procedimentos desenvolvidos aqui para testar simetria em tabelas multidimensionais.

- Obter medidas de evidência para homogeneidade marginal considerando classes de prioris mais gerais. Nesse caso, tais prioris podem ser aproximadas por misturas de distribuições Dirichlet.

- Investigar se existe alguma relação funcional entre a probabilidade a posteriori do teste de Jeffreys e a medida de evidência do FBST em testes de homogeneidade marginal.

- Reescrever o problema de homogeneidade marginal sob outras reparametrizações, como, por exemplo, através de transformações de variáveis aleatórias distribuídas segundo o modelo Gama. 


\section{Apêndice A}

\section{Derivação do p-valor para o teste de McNemar}

Resultado A.1 Suponha um vetor aleatório $\boldsymbol{X}=\left(n_{21}, n_{21}, n_{11}+n_{22}\right)$ que, fixado $\boldsymbol{\theta}=\left(\theta_{12}, \theta_{21}, \theta^{d}\right)$, possui distribuição Multinomial $(n ; \boldsymbol{\theta})$, onde $t=n_{12}+n_{21}$. O p-valor condicional, $p: \mathcal{X}^{\prime} \rightarrow[0,1]$, para testar $H: \theta_{12} \leq \theta_{21}$ contra $A: \theta_{12}>\theta_{21}$ baseado na estatística da razão de verossimilhanças generalizada condicional é, para cada $n_{12} \in \mathcal{X}^{\prime}$,

$$
p\left(n_{12}\right)= \begin{cases}P\left(\operatorname{Bin}\left(t, \frac{1}{2}\right) \geq n_{12}\right), & \text { se } n_{12}>t / 2 \\ 1, & \text { caso contrário }\end{cases}
$$

Demonstração:

Pela Equação 2.3, a função de verossimilhança gerada por $\boldsymbol{x}, V_{\boldsymbol{x}}$, pode ser fatorada em dois componentes ortogonais e, em termos de inferência sobre $\theta_{12}$ e $\theta_{21}$, podemos considerar apenas a distribuição condicional $n_{12} \mid t \sim \operatorname{Binomial}(t, \phi)$, onde $t=n_{12}+n_{21}$ e $\phi=\frac{\theta_{12}}{\theta_{12}+\theta_{21}}$. Portanto, o teste se reduz a $H^{\prime}: \phi \leq \frac{1}{2}$ contra $A^{\prime}: \phi>\frac{1}{2}$. Suponhamos que $n_{12} \leq t / 2$. Neste caso, definindo

$$
V_{n_{12} \mid t}^{\prime}(\phi)=\left(\begin{array}{c}
t \\
n_{12}
\end{array}\right) \phi^{n_{12}}(1-\phi)^{t-n_{12}}
$$

segue que

$$
\sup _{\phi \leq 1 / 2} V_{n_{12} \mid t}^{\prime}(\phi)=\sup _{0 \leq \phi \leq 1} V_{n_{12} \mid t}^{\prime}(\phi)
$$

fazendo com que $R V^{\prime}\left(n_{12}\right)=\frac{\sup _{\phi \leq 1 / 2} V_{n_{12} \mid t}^{\prime}(\phi)}{\sup _{0 \leq \phi \leq 1} V_{n_{12} \mid t}^{\prime}(\phi)}=1$. Neste caso,

$$
A_{n_{12}}=\left\{z \in \mathcal{X}^{\prime}: R V^{\prime}(z) \leq 1\right\}=\mathcal{X}^{\prime}
$$

Logo, para $0 \leq \phi \leq 1$ 


$$
p\left(n_{12}\right)=\sup _{\phi \leq 1 / 2} P_{\phi}\left(X \in \mathcal{X}^{\prime}\right)=1
$$

Assuma agora que $n_{12}>t / 2$. Assim,

$$
R V^{\prime}\left(n_{12}\right)=\frac{(t / 2)^{t}}{n_{12} n_{12}\left(t-n_{12}\right)^{t-n_{12}}} .
$$

Mas $f(x)=x^{x}(t-x)^{t-x}$ é uma função crescente para $x \geq t / 2$. Deste modo,

$$
\begin{aligned}
A_{n_{12}} & =\left\{z \in \mathcal{X}^{\prime}: R V^{\prime}(z) \leq R V^{\prime}(x)\right\} \\
& =\left\{m_{12} \in\{[t / 2]+1, \ldots, t\}: m_{12}^{m_{12}}\left(t-m_{12}\right)^{t-m_{12}} \geq n_{12} n_{12}\left(t-n_{12}\right)^{t-n_{12}}\right\} \\
& =\left\{m_{12} \in\{[t / 2]+1, \ldots, t\}: m_{12} \geq n_{12}\right\} .
\end{aligned}
$$

Por conseguinte, o p-valor é

$$
p\left(n_{12}\right)=P\left(\operatorname{Bin}(t, 1 / 2) \geq n_{12}\right) .
$$

Resultado A.2 Suponha um vetor aleatório $\boldsymbol{X}=\left(n_{12}, n_{21}, n_{11}+n_{22}\right)$ que, fixado $\boldsymbol{\theta}$, possui distribuição Multinomial $\left(n ;\left(\theta_{12}, \theta_{21}, \theta^{d}\right)\right)$, onde $t=n_{12}+n_{21}$. O p-valor condicional, $p: \mathcal{X}^{\prime} \rightarrow[0,1]$, para testar $H: \theta_{12}=\theta_{21}$ contra $A: \theta_{12} \neq \theta_{21}$ baseado na estatística da razão de verossimilhanças generalizada condicional é, para cada $n_{12} \in \mathcal{X}^{\prime}$,

$$
p^{*}\left(n_{12}\right)= \begin{cases}2 P\left(\operatorname{Bin}\left(t, \frac{1}{2}\right) \geq n_{12}\right), & \text { se } \quad n_{12} \geq t / 2 \\ 2 P\left(\operatorname{Bin}\left(t, \frac{1}{2}\right) \leq n_{12}\right), & \text { caso contrário, }\end{cases}
$$

Demonstração: Como $n_{12} \mid t \sim \operatorname{Binomial}(t, \phi)$, o teste se reduz a $H^{\prime}: \phi=\frac{1}{2}$ contra $A^{\prime}: \phi \neq \frac{1}{2}$. Temos que a estatística da razão de verossimilhanças condicional gerada por $n_{12}$ fica dada por

$$
R V^{\prime}\left(n_{12}\right)=\frac{\sup _{\phi=1 / 2} V_{n_{12} \mid t}^{\prime}(\phi)}{\sup _{0 \leq \phi \leq 1} V_{n_{12} \mid t}^{\prime}(\phi)}=\frac{\left(\frac{1}{2}\right)^{t}}{\left(\frac{n_{12}}{t}\right)^{n_{12}}\left(1-\frac{n_{12}}{t}\right)^{t-n_{12}}} .
$$

Note que

$\frac{\left(\frac{1}{2}\right)^{t}}{\left(\frac{n_{12}}{t}\right)^{n_{12}}\left(1-\frac{n_{12}}{t}\right)^{t-n_{12}}} \leq c \Leftrightarrow \frac{1}{\left(\frac{n_{12}}{t}\right)^{n_{12}}\left(1-\frac{n_{12}}{t}\right)^{t-n_{12}}} \leq c 2^{t} \Leftrightarrow\left(\frac{n_{12}}{t}\right)^{n_{12}}\left(1-\left(\frac{n_{12}}{t}\right)\right)^{t-n_{12}} \geq c^{*}$

em que $c^{*}=\left(c 2^{t}\right)^{-1}$. Consequentemente, 


$$
\begin{array}{r}
A_{n_{12}}=\left\{z \in \mathcal{X}^{\prime}: R V^{\prime}(z) \leq R V^{\prime}(x)\right\} \Leftrightarrow\left\{m_{12} \in\{0, \ldots, t\}:\left(\frac{n_{12}}{t}\right)^{n_{12}}\left(1-\left(\frac{n_{12}}{t}\right)\right)^{t-n_{12}}\right. \\
\left.\leq\left(\frac{m_{12}}{t}\right)^{m_{12}}\left(1-\left(\frac{m_{12}}{t}\right)\right)^{t-m_{12}}\right\} .
\end{array}
$$

Note que a função $g\left(n_{12}\right)=n_{12}^{n_{12}}\left(t-n_{12}\right)^{t-n_{12}}$ é simétrica em torno de $t / 2$, pois, dado $\delta>0$,

$$
\begin{aligned}
g(t / 2+\delta) & =\left(\frac{t / 2+\delta}{t}\right)^{t / 2+\delta}\left(1-\frac{(t / 2+\delta)}{t}\right)^{t-(t / 2+\delta)}=\left(\frac{t / 2+\delta}{t}\right)^{t / 2+\delta}\left(\frac{t / 2-\delta}{t}\right)^{t / 2-\delta} \\
& =g(t / 2-\delta) .
\end{aligned}
$$

Logo,

$$
A_{n_{12}}=\left\{m_{12} \in \mathbb{N}: m_{12} \geq n_{12}\right\} \cup\left\{m_{12} \in \mathbb{N}: m_{12} \leq t-n_{12}\right\}, \text { se } n_{12} \geq t / 2,
$$

E,

$$
A_{n_{12}}=\left\{m_{12} \in \mathbb{N}: m_{12} \leq n_{12}\right\} \cup\left\{m_{12} \in \mathbb{N}: m_{12} \geq t-n_{12}\right\} \text {, se } n_{12}<t / 2 .
$$

Assim, o p-valor fica dado por

$$
p^{*}\left(n_{12}\right)= \begin{cases}2 P\left(\operatorname{Bin}\left(t, \frac{1}{2}\right) \geq n_{12}\right), & \text { se } \quad n_{12} \geq t / 2 \\ 2 P\left(\operatorname{Bin}\left(t, \frac{1}{2}\right) \leq n_{12}\right), & \text { caso contrário. }\end{cases}
$$


APÊNDICE A 


\section{Apêndice B}

\section{Rotinas Computacionais}

\section{B FBST para Homogeneidade Marginal e Simetria em Tabelas Bidimensionais}

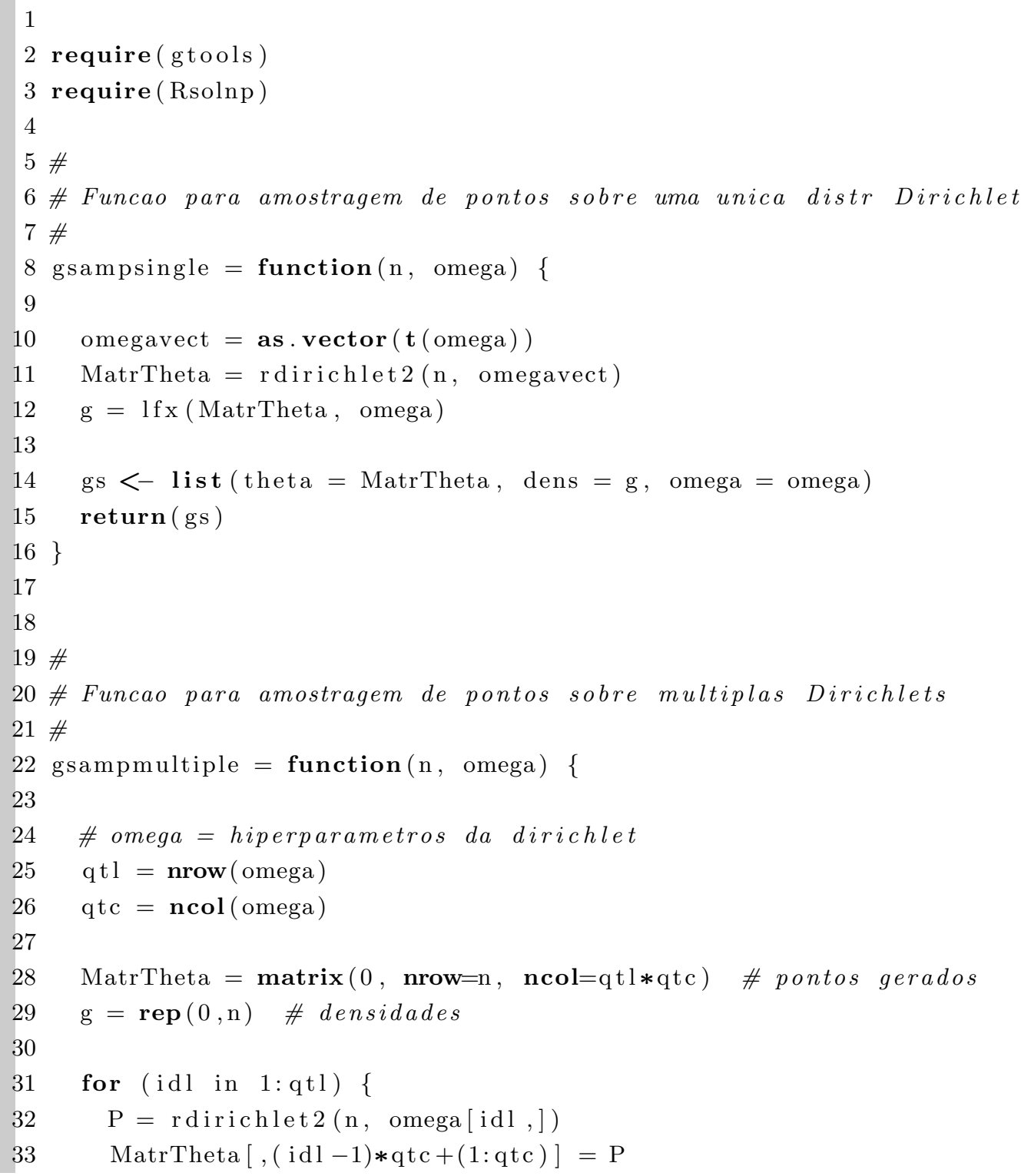




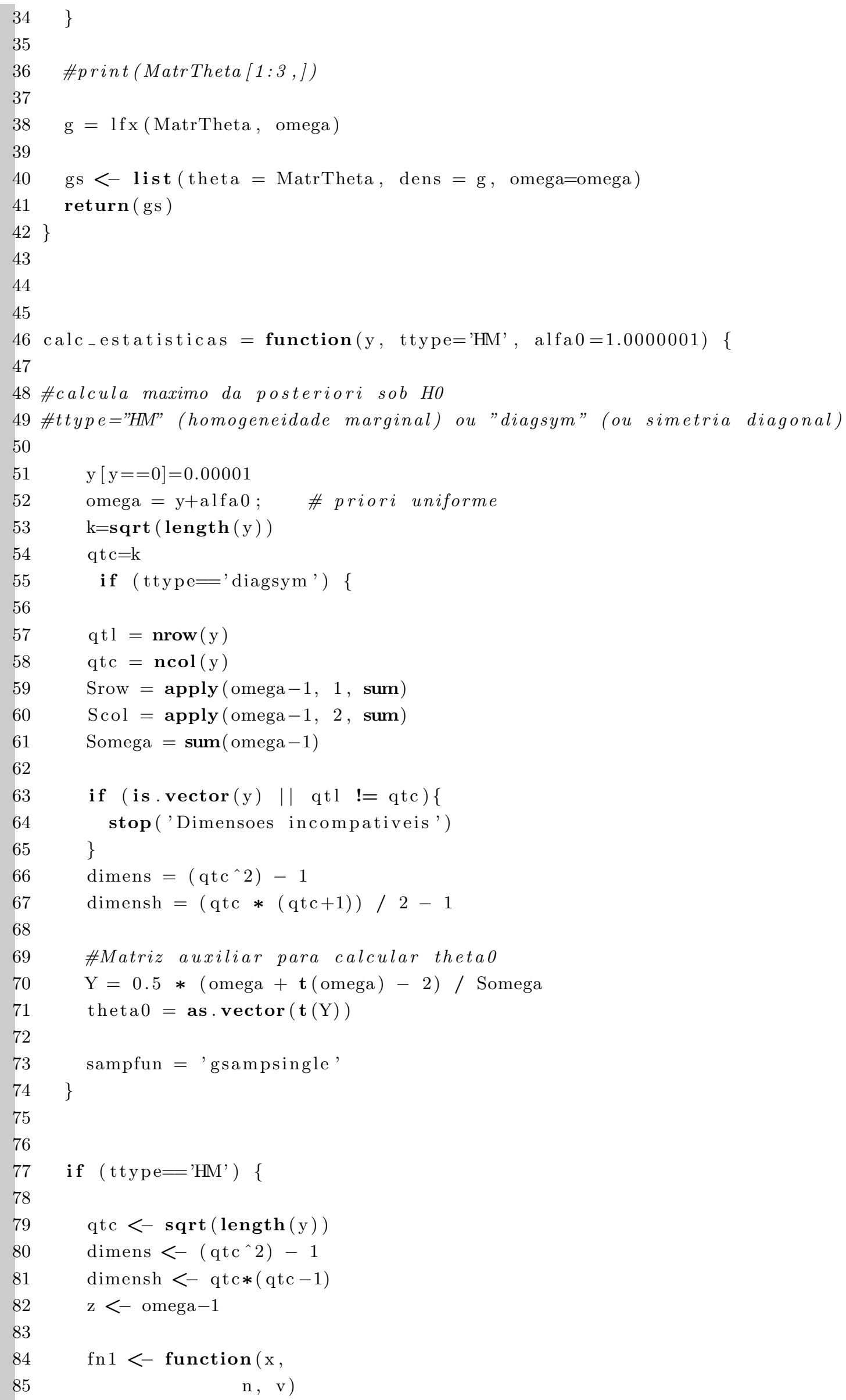




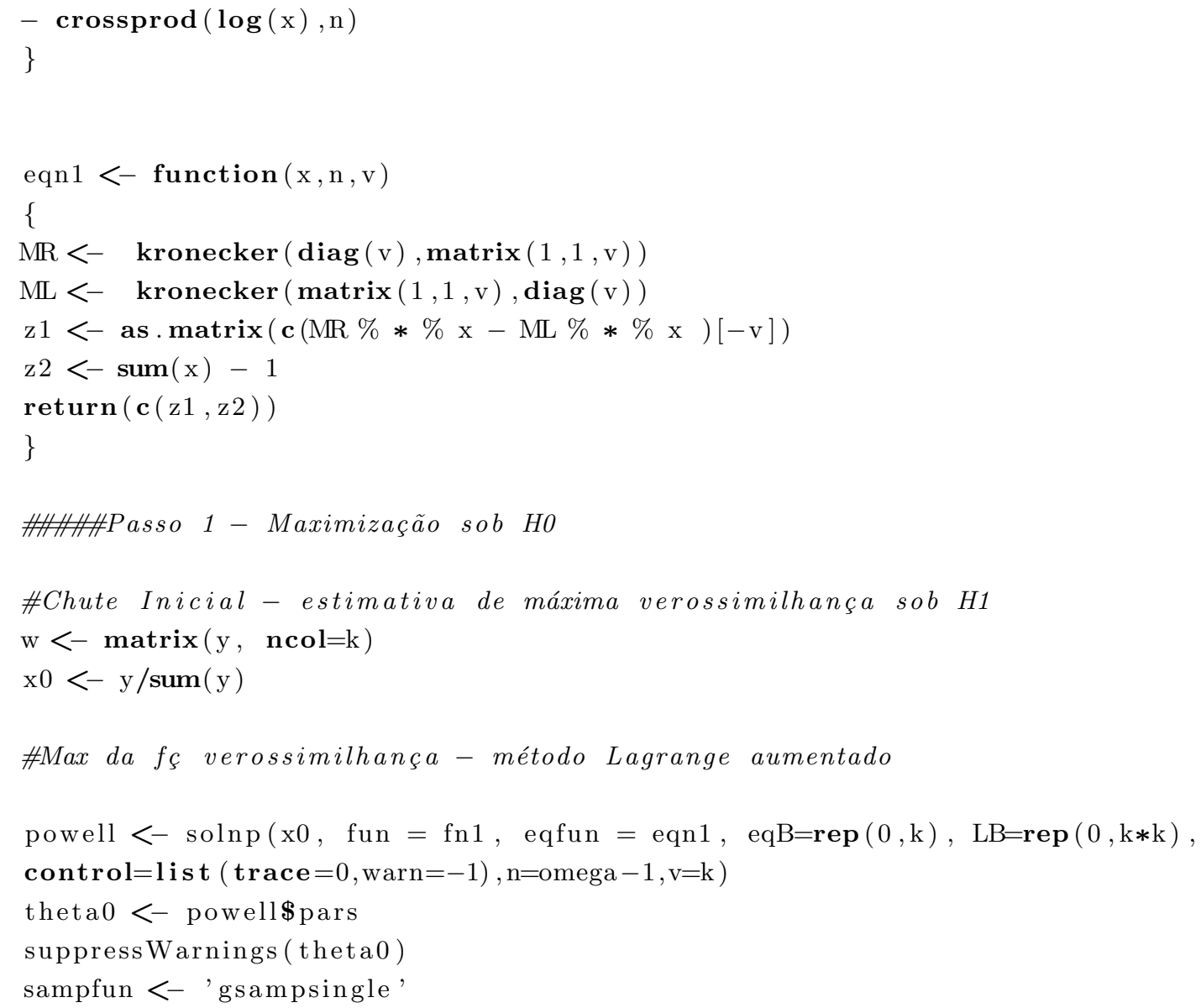


139 return ( list (evidH $=1-\mathrm{v}$, dimens=estat $\$$ dimens, dimensh=estat $\$$ dimensh, theta $0=$ estat $\$$ theta 0$))$

$140\}$

\section{Exemplo (página 17):}

$1 \mathrm{y}=\operatorname{scan}()$

$\begin{array}{llll}2 & 4 & 3 & 2\end{array}$

$\begin{array}{llll}3 & 1 & 5 & 8\end{array}$

$\begin{array}{lllll}4 & 7 & 3 & 4\end{array}$

5 evid.fbst (y, priori=c $(1,1,1,1), \quad n s i m=10000)$

\section{B FBST para Homogeneidade Marginal em Tabelas Tridimensi- onais}

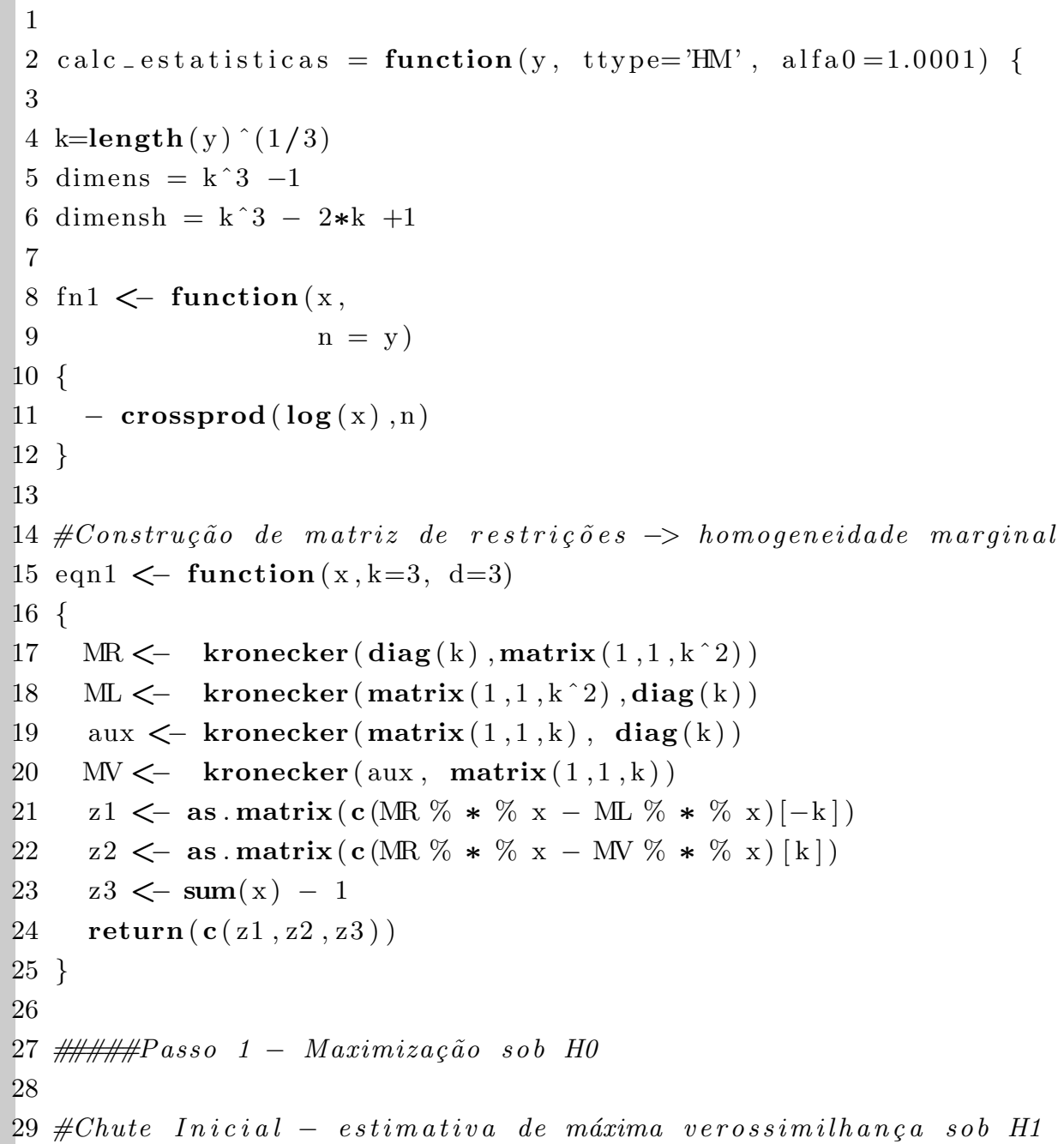




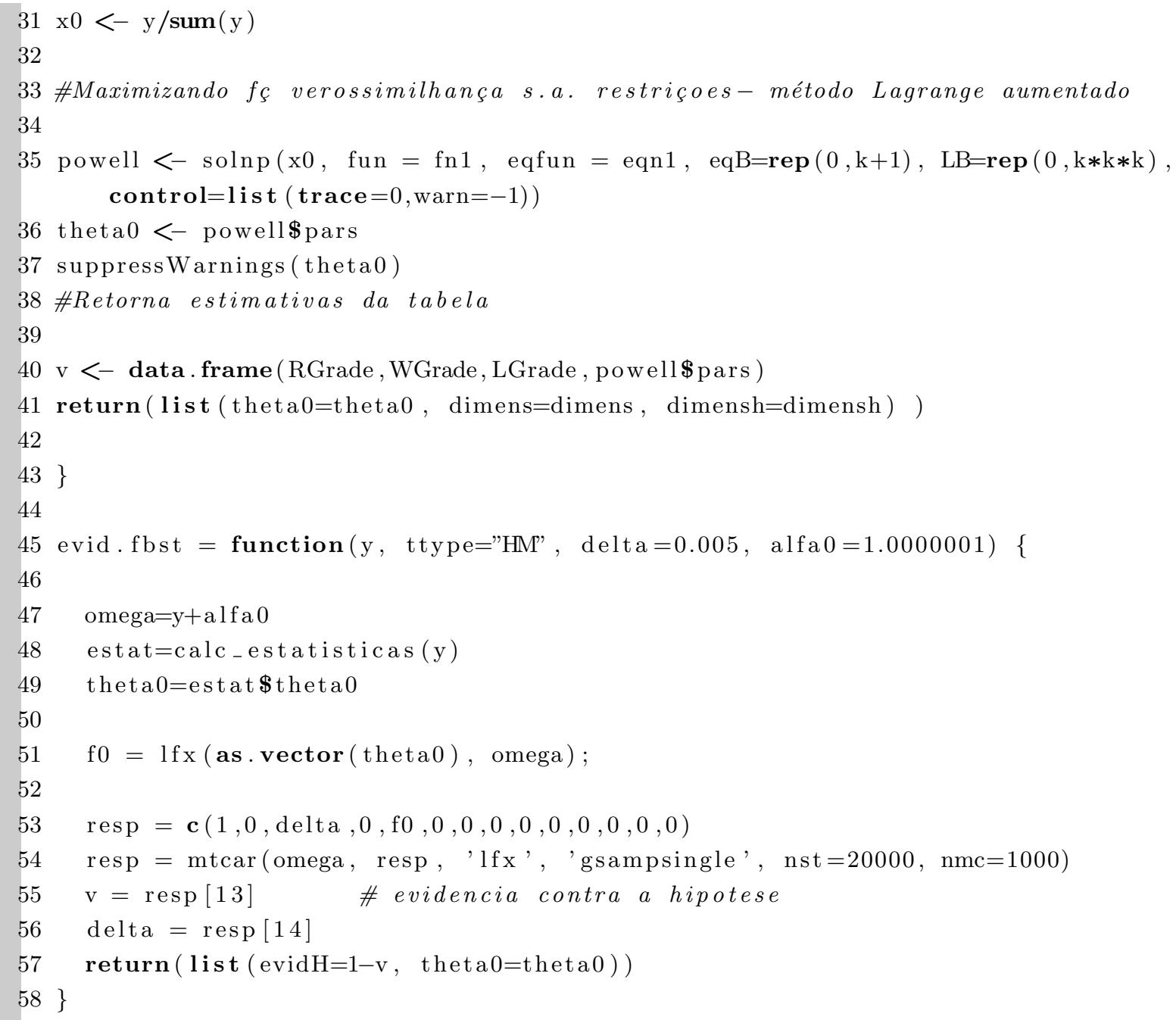


APÊNDICE B 


\section{Referências Bibliográficas}

Agresti (2007) Alan Agresti. An Introduction to Categorical Data Analysis. Wiley. Citado na pág. xvii, 26

Agresti (2013) Alan Agresti. Categorical Data Analysis. Wiley. Citado na pág. xvii, 1, 2, 7, 8, 9, 14,17

Aitchison e Shen (1980) J. Aitchison e S.M. Shen. Logistic-normal distributions: Some properties and uses. Biometrika. Citado na pág. 20, 23

Anderson (1951) T.W. Anderson. Probability models for analyzing time changes in attitudes. The Rand Corporation Research Memorandum. Citado na pág. xvii, 27, 28

Basso et al. (2009) Dario Basso, F. Pesarín, L. Salmaso e A. Solari. Permutation Tests for Stochastic Ordering and ANOVA: Theory and Applications with $R$. Springer-Verlag. Citado na pág. 19

Basu (1977) Debabrata Basu. On the elimination of nuisance parameters. Journal of the American Statistical Association. Citado na pág. 9

Berger e Delampady (1987) James Berger e Mohan Delampady. Testing precise hypothesis. Statistical Science. Citado na pág. 2

Berger e Wolpert (1988) James O. Berger e Robert L. Wolpert. The Likelihood Principle. Lecture Notes- Monograph Series. Citado na pág. 1

Bertsekas (1996) Dimitri P. Bertsekas. Contrained Optimization and Lagrange Multipliers Methods. Belmont, Massachusetts. Citado na pág. 15, 16

Bhapkar (1966) V.P. Bhapkar. A note on the equivalence of two test criteria for hypotheses in categorical data. Journal of the American Statistical Association. Citado na pág. 19

Bowker (1948) Albert H. Bowker. A test for symmetry in contingency tables. Journal of the American Statistical Association. Citado na pág. 14

Breen e Hayes (1997) Richard Breen e Bernadette Hayes. Religious mobility and party support in northern ireland. European Sociological Review. Citado na pág. xvii, 25, 26

Casella e Berger (2002) George Casella e Roger L. Berger. Statistical Inference. Duxburry Thomson Learning. Citado na pág. 2

Cox e Reid (1987) D. R. Cox e N. Reid. Parameter orthogonality and approximate conditional inference (with discussion). Journal The Royal Statistical Society. Citado na pág. 8

Davison e Hinkley (1997) A.C. Davison e D.V. Hinkley. Bootstrap Methods and Their Application. Cambridge Series on Statistical and Probabilistic Mathematics. Citado na pág. 19

DeGroot (1970) Morris H. DeGroot. Optimal Statistical Decisions. McGraw-Hill Book Company. Citado na pág. 2 
Diniz et al. (2012) Marcio A. Diniz, Carlos A.B. Pereira, Adriano Polpo, Julio M. Stern e Sergio Wechsler. Relationship between bayesian and frequentist significance indices. International Journal for Uncertainty Quantification. Citado na pág. 24

Fossaluza (2008) Victor Fossaluza. Testes de hipóteses em eleições majoritárias. Dissertação de Mestrado, Universidade de São Paulo. Citado na pág. 1

Gabriel (1969) K.R. Gabriel. Simultaneous test procedures - some theory of multiple comparisons. The Annals of Mathematical Statistics. Citado na pág. 17

Good (1956) Irving John Good. Good Thinking. University of Minnesota Press, Minneapolis, MN. Citado na pág. 4

Good (1976) Irving John Good. On the application of symmetric dirichlet distributions and their mixtures to contingency table. The Annals of Statistics. Citado na pág. 1

Good (2005) Philip I. Good. Permutation, Parametric and Bootstrap Tests of Hypotheses. Springer-Verlag, New York. Citado na pág. 19

Grizzle et al. (1969) James E. Grizzle, Frank Starmer e Gary G. Koch. Analysis of categorical data by linear models. Biometrics. Citado na pág. xvii, 30

Hastie et al. (2009) Trevor Hastie, Robert Tibshirani e Jerome Friedman. The Elements of Statistical Learning: Data Mining, Inference, and Prediction. Springer-Verlag New York. Citado na pág. 7

Hirji (2005) Karim F. Hirji. Exact Analysis of Discrete Data. Chapman \& Hall CRC. Citado na pág. 1

Holland (2007) Yvonne M. Bishop Stephen E. Fienberg Paul W. Holland. Discrete Multivariate Analysis. Springer-Verlag, New York. Citado na pág. 1

Irony et al. (2000) Telba Z. Irony, Carlos A.B. Pereira e Ram C. Tiwari. Analysis of opinion swing: Comparison of two correlated proportions. The American Statistician. Citado na pág. 1, 11

Izbicki (2010) Rafael Izbicki. Classes de testes de hipóteses. Dissertação de Mestrado, Universidade de São Paulo. Citado na pág. 17

Jeffreys (1961) Harrold Jeffreys. Theory of Probability. Oxford University Press. Citado na pág. 3, 11

Klingenberg e Agresti (2006) Bernhard Klingenberg e Alan Agresti. Multivariate extensions of mcnemar's test. Biometrics. Citado na pág. 7

Kullback (1959) S. Kullback. Information Theory and Statistics. John Wiley Sons. Citado na pág. 20

Lehmann e Romano (2005) E.L. Lehmann e Joseph P. Romano. Testing Statistical Hypotheses. Springer Science+Business Media, Inc, New York. Citado na pág. 18

Lindley (1964) Dennis V. Lindley. The bayesian analysis of contingency tables. The Annals of Mathematical Statistics. Citado na pág. 20

Madansky (1963) Albert Madansky. Tests of homogeneity for correlated samples. Journal of the American Statistical Association. Citado na pág. 19, 32

Madruga et al. (2001) M. Regina Madruga, Luis Gustavo Esteves e Sergio Wechsler. On the bayesianity of pereira-stern tests. Em Test. Citado na pág. 4 
Madruga et al. (2002) M.R. Madruga, Carlos A.B. Pereira e Julio Michael Stern. Bayesian evidence test for precise hypotheses. Journal of Statistical Planning and Inference. Citado na pág. $4,5,21$

McNemar (1947) Quinn McNemar. Note on the sampling error of the difference between correlated proportions or percentages. Psychometrika. Citado na pág. 1

Pace e Salvan (1997) L. Pace e A. Salvan. Principles of Statistical Inference. World ScientificSingapore, New York. Citado na pág. 9

Pereira e Viana (1982) Carlos A.B. Pereira e Marlos A.G. Viana. Elementos de Inferência Bayesiana. Citado na pág. 15

Pereira et al. (2008) Carlos A.B. Pereira, Julio M. Stern e Sergio Wechsler. Can a significance test be genuinely bayesian? Bayesian Analysis. Citado na pág. 4

Pereira e Stern (1999) Carlos B. Pereira e Julio Michael Stern. Evidence and credibility: Full bayesian significance test for precise hypotheses. Entropy. Citado na pág. 1, 4

Rodrigues (2006) Willian Waiteman Rodrigues. Testes de significância em tabelas de contingência 2x2 usando o modelo logístico-normal. Dissertação de Mestrado, Universidade de São Paulo. Citado na pág. 20

Schervish (1995) Mark J. Schervish. Theory of Statistics. Springer-Verlag New York. Citado na pág. 3,14

Schervish (1996) Mark J. Schervish. P values: What they are and what they are not. The American Statistician. Citado na pág. 10

Schervish (1999) Michael Lavine Mark J. Schervish. Bayes factors: What they are and what they are not. The American Statistician. Citado na pág. 1

Sidik (2001) Roger L. Berger Kurex Sidik. Exact unconditional tests for 2x2 matched-pairs design. Relatório técnico, North Carolina State University. Citado na pág. 1

Silvapulle e Sen (2005) Mervyn J. Silvapulle e Pranab K. Sen. Constrained Statistical Inference. Wiley. Citado na pág. 9

Stuart (1953) Alan Stuart. The estimation and comparison of strenghts of association in contingency tables. London School of Economics and Political Science. Citado na pág. xvii, 13, 14, 19

Ziegler e König (2010) Andreas Ziegler e Inke R. König. A Statistical Approach to Genetic Epidemiology. Wiley. Citado na pág. 7 